\title{
A human brain vascular atlas reveals diverse mediators of Alzheimer's risk
}

https://doi.org/10.1038/s41586-021-04369-3

Received: 23 April 2021

Accepted: 17 December 2021

Published online: 14 February 2022

Check for updates

\author{
Andrew C. Yang ${ }^{1,2,3 凶}$, Ryan T. Vest ${ }^{3,13}$, Fabian Kern ${ }^{3,4,13}$, Davis P. Lee ${ }^{3}$, Maayan Agam³, \\ Christina A. Maat ${ }^{3}$, Patricia M. Losada ${ }^{3}$, Michelle B. Chen ${ }^{1}$, Nicholas Schaum ${ }^{3}$, \\ Nathalie Khoury ${ }^{3}$, Angus Toland ${ }^{5}$, Kruti Calcuttawala ${ }^{3}$, Heather Shin ${ }^{3}$, Róbert Pálovics ${ }^{3}$, \\ Andrew Shin ${ }^{3}$, Elizabeth Y. Wang ${ }^{6}$, Jian Luo ${ }^{7}$, David Gate ${ }^{3}$, Walter J. Schulz-Schaeffer ${ }^{8}$, \\ Pauline Chu ${ }^{4}$, Julie A. Siegenthaler ${ }^{9}$, M. Windy McNerney ${ }^{10}$, Andreas Keller ${ }^{3,4}$ \& \\ Tony Wyss-Coray ${ }^{2,3,11,12 \llbracket}$
}

The human brain vasculature is of great medical importance: its dysfunction causes disability and death ${ }^{1}$, and the specialized structure it forms-the blood-brain barrierimpedes the treatment of nearly all brain disorders ${ }^{2,3}$. Yet so far, we have no molecular map of the human brain vasculature. Here we develop vessel isolation and nuclei extraction for sequencing (VINE-seq) to profile the major vascular and perivascular cell types of the human brain through 143,793 single-nucleus transcriptomes from 25 hippocampus and cortex samples of 9 individuals with Alzheimer's disease and 8 individuals with no cognitive impairment. We identify brain-region- and species-enriched genes and pathways. We reveal molecular principles of human arteriovenous organization, recapitulating a gradual endothelial and punctuated mural cell continuum. We discover two subtypes of human pericytes, marked by solute transport and extracellular matrix (ECM) organization; and define perivascular versus meningeal fibroblast specialization. In Alzheimer's disease, we observe selective vulnerability of ECM-maintaining pericytes and gene expression patterns that implicate dysregulated blood flow. With an expanded survey of brain cell types, we find that 30 of the top 45 genes that have been linked to Alzheimer's disease risk by genome-wide association studies (GWASs) are expressed in the human brain vasculature, and we confirm this by immunostaining. Vascular GWAS genes map to endothelial protein transport, adaptive immune and ECM pathways. Many are microglia-specific in mice, suggesting a partial evolutionary transfer of Alzheimer's disease risk. Our work uncovers the molecular basis of the human brain vasculature, which will inform our understanding of overall brain health, disease and therapy.
Brain health depends on brain vascular health. The brain is one of the most highly perfused organs in the body, which is necessary to meet its unique metabolic needs ${ }^{4}$. Brain vascular dysfunction contributes to stroke ${ }^{1}$, congenital neurological disorders ${ }^{5}$ and age-related neurodegenerative disease $^{5,6}$. The brain vasculature forms a special structure-the blood-brain barrier (BBB)-that mediates selective and haemodynamically responsive movement of molecules between the blood and the brain ${ }^{2,3}$. Although necessary for optimal neuronal function ${ }^{4}$, the BBB frustrates the pharmacological treatment of nearly all brain disorders ${ }^{7,8}$, and extensive efforts are underway to discover targets on the human BBB to enhance drug delivery.

Specialized brain vascular properties arise from a complex community of interacting cell types ${ }^{9-11}:$ endothelial cells, adjacent mural smooth muscle cells (SMCs) and pericytes, perivascular immune cells, and surrounding astrocytes that differ across brain regions and vary along an arteriovenous gradient ${ }^{12}$. Heterogeneity along this gradient produces functionally segmented circulatory, metabolic and permeability properties that are necessary for brain health ${ }^{3,5}$.

Previous work has profiled the mouse brain vasculature ${ }^{12-15}$, but it remains unclear how conserved these findings are in humans. Single-nucleus studies have elucidated the cellular heterogeneity of the human brain in health and disease ${ }^{16-19}$. However, although vascular cell density ${ }^{20,21}\left(70,000\right.$ cells per $\left.\mathrm{mm}^{3}\right)$ approaches total glia density ${ }^{20}$, previous studies have depleted human brain vascular cells for unknown reasons. Although human brain microvessels can be isolated for bulk

'Department of Anatomy, University of California San Francisco, San Francisco, CA, USA. ${ }^{2}$ Bakar Aging Research Institute, University of California San Francisco, San Francisco, CA, USA. ${ }^{3}$ Department of Neurology and Neurological Sciences, Stanford University School of Medicine, Stanford, CA, USA. ${ }^{4}$ Chair for Clinical Bioinformatics, Saarland University, Saarbrücken, Germany. ${ }^{5}$ Department of Pathology, Stanford University School of Medicine, Stanford, CA, USA. ${ }^{6}$ Department of Surgery, Icahn School of Medicine at Mount Sinai, New York, NY, USA. ${ }^{7}$ Veterans Administration Palo Alto Healthcare System, Palo Alto, CA, USA. ${ }^{8}$ Institute for Neuropathology, Saarland University Hospital and Medical Faculty of Saarland University, Homburg, Germany. ${ }^{9}$ Department of Pediatrics, University of Colorado Anschutz Medical Campus, Aurora, CO, USA. ${ }^{10}$ Department of Psychiatry, Stanford University School of Medicine, Stanford, CA, USA. "Wu Tsai Neurosciences Institute, Stanford University, Stanford, CA, USA. ${ }^{12}$ Paul F. Glenn Center for the Biology of Aging, Stanford University School of Medicine, Stanford, CA, USA.

${ }^{13}$ These authors contributed equally: Ryan T. Vest, Fabian Kern. ${ }^{凶}$ e-mail: andrew.yang@ucsf.edu; twc@stanford.edu 

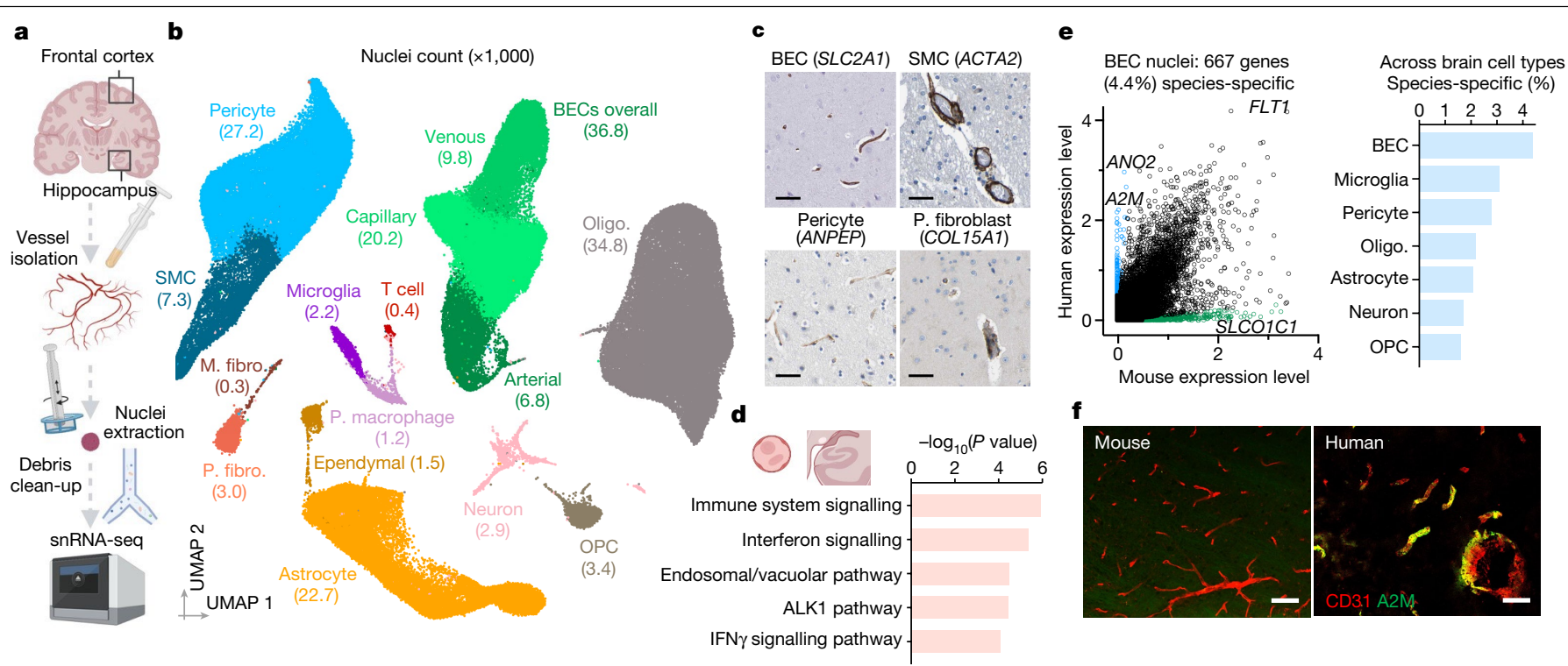

Fig. $1 \mid$ Cells of the human brain vasculature. a, VINE-seq method to enrich vascular nuclei from post-mortem human brain samples. b, Enrichment of vascular and perivascular cell types from the human cortex and hippocampus. UMAP of 143,793 nuclei from 25 human hippocampus and superior frontal cortex samples across 17 individuals, coloured by cell type and labelled with numbers of nuclei. M. fibro., meningeal fibroblast; oligo., oligodendrocyte; OPC, oligodendrocyte precursor cell; P. fibro., perivascular fibroblast; P. macrophage, perivascular macrophage.c, Immunohistochemical validation of cell-type-specific gene markers. Scale bars, $50 \mu \mathrm{m}$. Image credit: Human Protein Atlas ${ }^{25}$ (http://www.proteinatlas.org). d, Enriched biological pathways in BECs from the hippocampus compared to the superior frontal cortex, in individuals with $\mathrm{NCI}(P<0.05$, cumulative hypergeometric test). e, Left, scatter plot depicting mRNA expression levels ( $\log C P M)$ of mouse and human genes with one-to-one orthologues in BECs. Divergently expressed genes are coloured ( $>10$-fold difference, minimum $0.5 \log _{2}$ CPM expression). Right, proportion of the transcriptome of each brain cell type that is specific to human versus mouse (same thresholds). f, Immunohistochemical validation of A2M protein specifically in the human but not mouse vasculature. Scale bars, $50 \mu \mathrm{m}$. assays, at present no method exists-to our knowledge-to extract nuclei from microvessels for analysis at single-cell resolution.

Given the scientific, medical and pharmacological importance of the human brain vasculature, we set out to create a single-cell atlas of the human brain vasculature of the hippocampus and cortex on the basis of samples from individuals with no cognitive impairment $(\mathrm{NCI})$ and from individuals with Alzheimer's disease (AD).

\section{Cells of the human brain vasculature}

Extracting nuclei from brain microvessels is challenging because the method must dissociate the vessel basement membrane to release nuclei without damaging the nuclei themselves. We systematically explored enzymatic, chemical and physical approaches. Most resulted in damaged nuclei devoid of RNA reads. We found success in adapting a gentle protocol for splenocyte isolation (Methods), and combined it with extensive clean-up using sucrose and fluorescence-activated cell sorting (FACS) to ensure high-quality data (Fig. 1a, Extended Data Fig. 1). We used our method, VINE-seq (vessel isolation and nuclei extraction for sequencing), to process 25 samples: the hippocampus of 9 individuals with AD and 8 age- and sex-matched individuals with $\mathrm{NCI}$, as well as the superior frontal cortex from a subset of 8 individuals (4 per group) (Supplementary Table 1). Samples included a range of $A P O E$ genotypes, and NCI samples exhibited no significant vascular pathologies in the studied hippocampus and cortex tissue (Supplementary Table 1, Extended Data Fig. 2a). After quality control (Methods), we obtained 143,793 single-nucleus transcriptomes. Visualization in uniform manifold approximation and projection (UMAP) space separated nuclei into 15 major cell types (Fig. 1b), including all known vascular and perivascular cell types: endothelial cells (arterial, capillary and venous), SMCs, pericytes, astrocytes, macrophages, T cells, and both perivascular and meningeal fibroblasts. The number of cerebrovascular nuclei captured here exceeds those in the literature by several hundred-fold
(Supplementary Table 2, Extended Data Fig. 2b, c). Vascular cell-type markers were validated in situ (Fig. 1c). Data can be browsed at https:// twc-stanford.shinyapps.io/human_bbb.

VINE-seq captured three types of immune cell in the human brain: microglia, macrophages and T cells. Over 300 genes differentiated brain border macrophages and microglia, yielding new markers for human studies (Extended Data Fig. 2d-f). Analysis of canonical markers indicated our capture of brain memory CD8 cytotoxic and CD4 T cells (Extended Data Fig. 2g). Brain regional transcriptome differences were evident (Supplementary Table 3). Astrocyte transcriptional identity was the most influenced by brain region (Extended Data Fig. $2 \mathrm{~h}-\mathrm{k}$ ). Moreover, hippocampal endothelial cells showed greater baseline inflammation-indicated for example by higher levels of interferon- $\gamma$ (IFN $\gamma$ ) signalling-than did those in the cortex (Fig. 1d). Such inflammatory signalling may inhibit hippocampal neurogenesis, and, together with accelerated hippocampal pericyte loss (Extended Data Fig. 1h), provides a molecular hypothesis for the vulnerability and dysfunction of the hippocampal vasculature ${ }^{22}$.

We next compared nuclei transcriptomes between human and mouse endothelial cells and pericytes. Using a strict cut-off $(>10 \times$ difference, log-transformed counts per million (CPM) > 0.5) (Supplementary Table 4), we found hundreds of species-enriched genes in brain endothelial cells (BECs) and pericytes (Fig.1e). Understanding species differences in neurons and glia has been a subject of intense study. Together with microglia ${ }^{23}$, we find that BECs and pericytes exhibit the greatest transcriptional divergence (Fig. 1e, Extended Data Fig. 3). Several vascular solute transporters (for example, the $\gamma$-aminobutyric acid (GABA) transporter encoded by SLC6A12) vary by species, which suggests differences in brain metabolism (Extended Data Fig. 3g). Several genes of disease and pharmacological importance also vary (Extended Data Figs. 3, 4). Together, the VINE-seq method opens the human brain vasculature for molecular study and provides a data resource for investigating its diverse cell types. 

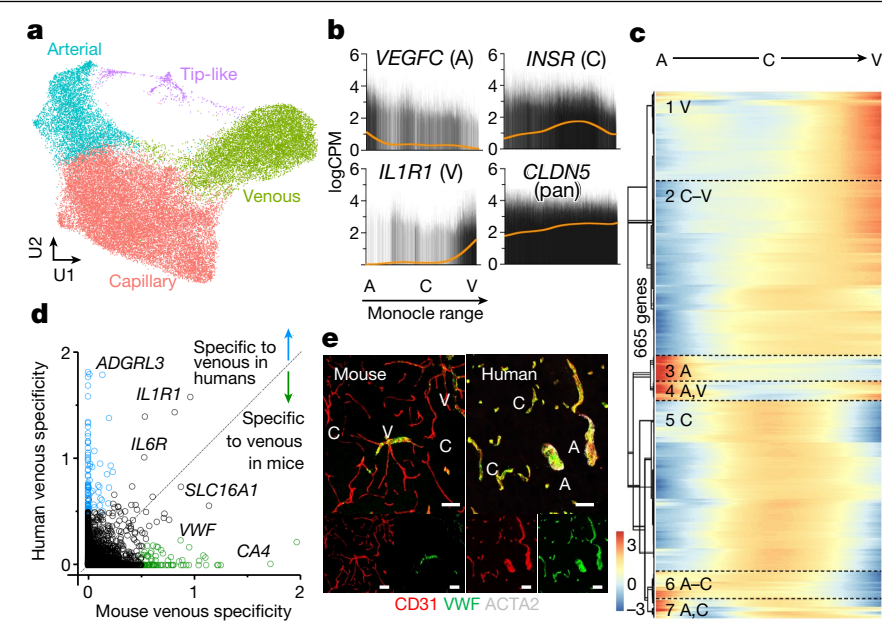

Fig. 2 Organizing principles of human BECs. a, UMAP of 36, 825 human BEC nuclei, coloured by zonation. $\mathbf{b}$, Zonal expression of transcripts across human BECs ordered by Monocle pseudotime. LOWESS regression line (orange) and density of black lines (counts) correspond with expression levels. A, arterial; $\mathrm{C}$, capillary; $\mathrm{V}$, venous. c, Heat map of zonation-dependent gene expression in human BECs $(36,825$ nuclei). d, Scatter plot depicting the specificity of transcripts for venous BECs in mice ${ }^{12}$ versus humans. Venous specificity score $=$ average $(\operatorname{logFC}($ vein/capillary), $\operatorname{logFC}($ vein/artery) $)$ (in which FC refers to fold change). For example, $V W F$ is predicted to be more specific to venous $\mathrm{BECs}$ in mice than it is in humans. See Extended Data Fig. 9 for arterial and capillary specificity plots. e, Immunohistochemical validation of VWF specificity to venous BECs in mice but not in humans. Scale bars, $50 \mu \mathrm{m}$.

\section{Endothelial and mural cell organization}

With our large-scale capture of vascular nuclei (more than 30 times the number previously reported in mice $^{12}$ and more than 200 times that previously reported in humans, to our knowledge ${ }^{16,17}$ ), we sought to comprehensively characterize the molecular basis of endothelial and mural cell organization along the arteriovenous axis of the human brain vasculature. Changes along this axis are referred to as zonation ${ }^{12,15}$. Beginning with BECs, we clustered 36,825 nuclei into known arterial, capillary and venous segments (Fig. 2a, Extended Data Fig. 5). Clusters were defined by established zonation markers, such as arterial VEGFC and $A L P L$; capillary MFSD2A and SLC7A5; and venous IL1R1 and $N R 2 F 2^{12,15}$. Although capillaries represent most (around 90\%) of the endothelium, VINE-seq captured rarer arterial (at 19\%) and venous (27\%) BECs, because of better strainer retention or nuclei liberation. We noticed a small cluster ( 571 nuclei, around $0.1 \%$ ) outside conventional zonation. This cluster expressed genes characteristic of 'tip' cells (for example, PLAUR and $L A M B 1$ ) as well as 'proteostatic' heat shock proteins.

We next ordered endothelial nuclei along a one-dimensional pseudotime ${ }^{24}$ axis to recapitulate the anatomical arteriovenous axis. Arterial and venous markers peaked at opposite ends, with capillary markers in between (Fig. 2b). We used the 665 most significantly variable cluster genes to unbiasedly order endothelial nuclei and observed 7 gradually changing patterns of gene expression, representing arterial, capillary or venous markers, and combinations thereof (Fig. 2c). We confirmed that the Monocle range represented a cell order matching anatomical arteriovenous zonation through immunostaining ${ }^{25}$ (Extended Data Fig. 6a). Our patterns recapitulate the gradual zonation continuum described in mice-but, notably, this similar overall continuum arises from significantly different component zonation markers (Supplementary Tables 2,5).

We thus wondered whether established zonation markers in mice would be conserved in humans. We calculated a score (Methods) measuring the specificity of each gene to a given zonation (for example, arterial, capillary or venous). Indeed, we observed in each vessel segment a significant number of markers that lost their zonation specificity between species (Fig. 2d, Extended Data Fig. 6). For example, the blood clotting gene von Willebrand factor $(V W F)$ is largely expressed in mouse venous endothelial cells ${ }^{12,15}$. However, $V W F$ is expressed throughout the human cerebrovasculature, even in small-diameter capillaries(Fig.2d,e). VWF abundance predisposes for ischaemic stroke ${ }^{26}$, which raises implications for translational studies of stroke.

Next, with mural cells, we clustered 34,508 nuclei into arterial smooth muscle cells (aSMCs), arteriolar SMCs (aaSMCs), and notably, two subclusters of pericytes (Fig. 3a, b). One pericyte subcluster enriched for small-molecule transmembrane transport, which we call T-pericytes (for transport), whereas the other subcluster enriched for extracellular matrix (ECM) organization, (M-pericytes, for matrix). Thus, function rather than anatomical location may be the major driver of human pericyte transcriptional identity. M-pericytes may therefore contribute to small-vessel diseases such as CADASIL (cerebral autosomal dominant arteriopathy with subcortical infarcts and leukoencephalopathy), CARASIL (cerebral autosomal recessive arteriopathy with subcortical infarcts and leukoencephalopathy) and collagen IV deficiencies for which perturbations in the cerebrovascular ECM cause disease ${ }^{27}$. Moreover, human T-pericytes-but not mouse pericytes-express transporters like the GABA transporter $S L C 6 A 1$ (involved in epilepsy) and the glutamate transporter SLC1A3 (Extended Data Fig. 3g). Because recent mouse pericyte datasets ${ }^{12}$ have cautioned against endothelial contamination, we assessed and found no such contamination in our human pericyte nuclei (Extended Data Fig. 2c).

To study mural cell zonation, we used the 670 most significantly variable and robustly expressed cluster genes to order all mural cell nuclei and observed the expected order of aSMC markers on one end (for example, $A C T A 2$ and $T A G L N$ ), followed by aaSMC (for example, SLIT3 and CTNNA3); and pericyte markers on the other end (for example, $A B C C$ 9 and $P T N$ ), confirmed in situ (Fig. 3c, Extended Data Fig. 6d). Recapitulating patterns described in mice ${ }^{12}-$ and as opposed to the gradual zonation pattern in BECs-, human mural cells exhibited an abrupt gene expression transition between SMCs and pericytes (Fig. 3c). To determine whether the ordering of T- and M-pericytes (Fig. $3 \mathrm{c}$ ) reflects anatomical arteriovenous order, we mapped them to mouse mural cell markers ${ }^{12}$ and found no differences in their expression of mouse capillary 'pericyte' versus venous mural cell 'vSMC' markers (Fig. 3d). This suggests that $\mathrm{T}$ - and $\mathrm{M}$-pericytes do not segregate by arteriovenous segment. Immunostaining confirmed that $\mathrm{T}$ - and $\mathrm{M}$-pericyte markers are each present across both small- and large-diameter brain vessels (Extended Data Fig. 6d).

Together, these data suggest that human pericytes transcriptionally identify more by functional specialization than by arteriovenous location; and that whereas aSMCs and aaSMCs reside on arterial and arteriolar vessels, respectively, $\mathrm{T}$ - and $\mathrm{M}$-pericytes intermix along the capillary and venous vasculature. As with BECs, we find that only a minority of top mouse SMC and pericyte markers retain their predictive value in humans (Fig. 3e, Extended Data Figs. 3b, 6d). Because proteins encoded by zonation marker genes perform a variety of key functions at defined arteriovenous locations, species-specific endothelial and mural cell differences are likely to reflect fundamental differences in brain vascular properties that can now be studied.

\section{Brain fibroblasts molecularly defined}

Using annotations from previous mouse studies ${ }^{12,28}$, we noticed fibroblasts from both the vascular and the meningeal barriers of the human brain (Fig. 4a). Cooperating with the vascular BBB, the recently (re) discovered meningeal lymphatics has important roles in waste clearance and neuroimmune surveillance ${ }^{29,30}$. Fibroblasts transcriptionally segregated according to anatomical location: vascular versus meningeal (Fig. 4a, b); but they also separated according to meningeal layers (Extended Data Fig. 7a, b). Pathway enrichment analysis of marker 


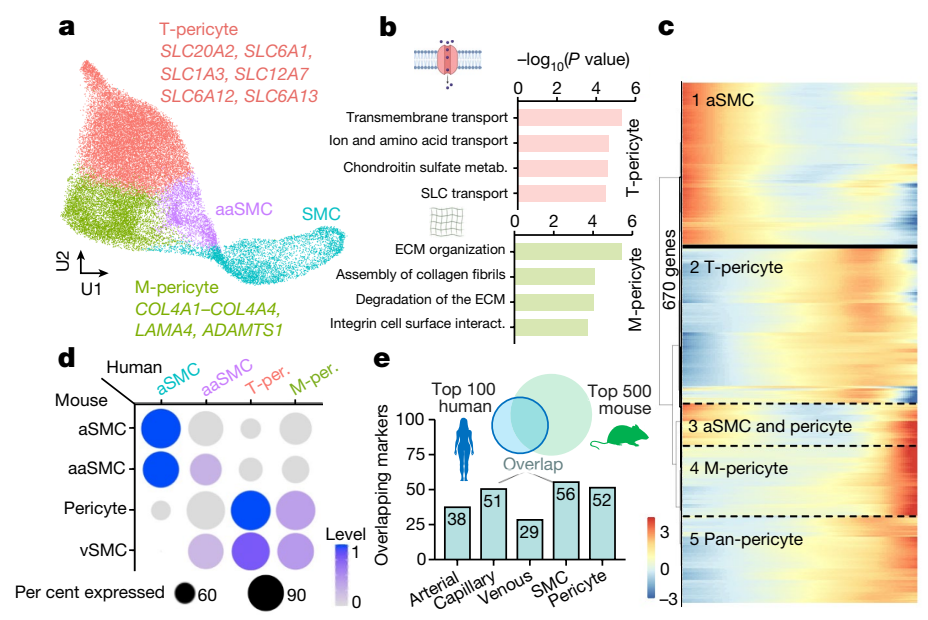

Fig. 3 Organizing principles of human brain mural cells. a, UMAP of 34,508 human pericyte and SMC nuclei, coloured by cell subtype.b. Enriched biological pathways in $\mathrm{T}$ - and $\mathrm{M}$-pericytes compared to the remaining SMC and pericyte populations $(P<0.05$, cumulative hypergeometric test). c, Heat map of gene expression in human SMCs and pericytes (34,508 human mural nuclei). Solid line delineating aaSMCs and aSMCs from pericytes reflects an abrupt transcriptomic transition. Unlike BECs, mural cell ordering does not reflect anatomical arteriovenous ordering. d, Mapping the expression of mouse mural cell markers onto human mural cell types. The top 500 mouse markers were aggregated into four distinct modules ('aSMCs', 'aaSMCs', capillary 'pericytes' and venous smooth muscle cells ' $\left.v S M C s^{\prime}\right)^{12}$ and their expression was assessed in the four transcriptionally distinct human mural cell types. e, Overlap between the top 100 human endothelial and mural cell subtype markers and those identified in mice. A more lenient set of 500 mouse markers ${ }^{12}$ was used for comparison for robust results. Species conservation of a cell-type marker depends both on species-specific changes in the given cell type and on changes among the remaining cell types. genes revealed specialized fibroblast functions (Fig. 4b): perivascular fibroblasts showed enriched expression for ECM structural components or its modifiers or receptors (for example, 'TGF $\beta$ regulation of $E C M^{\prime}$ ), whereas meningeal fibroblasts enriched for solute transporters. Fibrotic scars arise from the pathological deposition of collagen I (COL1)-rich ECM. Consistent with mouse lineage-tracing studies ${ }^{31}$, our work indicates that human perivascular fibroblasts are the main producers of COL1 in the brain and thus are likely to form fibrotic scars after brain injury (Extended Data Fig. 7c).

Closer comparison of differentially expressed genes (DEGs) between fibroblast populations revealed a polarization of solute influx and efflux pumps: meningeal fibroblasts specifically expressed SLC influx solute transporters, whereas perivascular fibroblasts exclusively expressed ABC efflux pumps (Fig. 4d). We confirmed this polarized expression in situ (Fig. 4e, Extended Data Fig. 7d, e). Perivascular fibroblasts reside in the Virchow-Robin space, and thus like meningeal fibroblasts, bathe in the cerebrospinal fluid (CSF). This cooperative circuit of polarized transporters suggests fibroblast regulation of solute exchange between the brain and the $\mathrm{CSF}^{32}$. Finally, as in endothelial and mural cells, perivascular fibroblast-like cell markers varied by species (Extended Data Fig. 7f). Together, these data provide a characterization of human brain fibroblast diversity, revealing the molecular basis of their anatomical specialization and a cooperative circuit for CSF solute exchange.

\section{Vascular cell-type perturbations in AD}

$\mathrm{AD}$ is a neurodegenerative disorder with progressive impairment of cognitive function leading to dementia. Impairment arises from complex perturbations in cell composition and gene expression. We thus sought to profile changes in the AD human brain vasculature at single-cell resolution. We defined our patient groups by clinical diagnosis and confirmed the presence of $\beta$-amyloid plaques in the hippocampus and cortex (Extended Data Fig. $8 \mathrm{a}-\mathrm{c}$ ).

Previous studies have identified context-dependent, diseaseassociated glial subpopulations ${ }^{16,17,33}$. We did not observe the emergence of new vascular cell subclusters in AD (Fig. 5a, Extended Data Fig. 8d). By contrast, we found a strong loss of brain vascular nuclei-across endothelial cells, SMCs, pericytes and fibroblast-like cells (Fig. 5a, b). Thus, beyond focal cerebrovascular damage, vascular loss may be widespread across the arteriovenous axis. Among pericytes, M-pericytes involved in ECM organization exhibited selective vulnerability (Fig. 5b), providing a molecular hypothesis for the structural breakdown of the $\mathrm{BBB}$ that is reported in $\mathrm{AD}^{22}$. However, FACS and single-nucleus RNA sequencing (snRNA-seq) approaches may not always yield reliable cell quantifications. Thus, we stained hippocampal tissue to quantify the number of Hoechst ${ }^{+}$vascular cells within collagen IV $^{+}$cerebrovasculature. Although we found no significant differences in the area of collagen $\mathrm{IV}^{+}$vessels or in the total number of Hoechst ${ }^{+}$nuclei, we found a significant decrease with $\mathrm{AD}$ in the number of $\mathrm{Hoechst}^{+}$nuclei within collagen $\mathrm{IV}^{+}$vessels, consistent with reports of 'string vessels' in disease $^{34}$ (Fig. 5b, Extended Data Fig. 8d, e). In short, we find selective vulnerability of specific vascular cell subpopulations in AD.

We next examined cell-type-specific changes in gene expression in $\mathrm{AD}$ (Methods). We identified 463 unique DEGs using more stringent thresholds (Methods, Fig. 5c, Supplementary Table 6). Overall, mural cells exhibited the strongest changes, and other cell types exhibited a signature of gene repression: 61-78\% of DEGs were downregulated (Fig. 5c). DEGs were robustly detected across different levels of expression (Supplementary Table 6). Most DEGs were cell-type- and zonation-specific (Extended Data Fig. 8f), suggesting a heterogenous response to AD pathology across the vasculature. Notably, several DEGs are riskgenes implicated in GWASs of AD and small-vessel disease (Extended Data Fig. $8 \mathrm{~g}, \mathrm{~h}$ ). At the pathway level, DEGs in mural cells and fibroblasts implicated dysregulated vasoconstriction and compromised blood flow (Fig. 5d). This provides a potential molecular mechanism for the cerebral hypoperfusion that is discernible in magnetic resonance imaging (MRI) scans of patients with $\mathrm{AD}^{35}$.

Carriers of the $A P O E 4$ allele may exhibit accelerated breakdown of the BBB before cognitive impairment ${ }^{36}$. We found marked interferon inflammation in the endothelium of $A P O E 4$ carriers (Fig. 5e, Extended Data Fig. 8i-k, Supplementary Table 7). Next, we compared AD DEGs in human BECs with those in mouse models of AD. Such models have facilitated mechanistic study of $\beta$-amyloid pathology, but recent reports describe significant species differences in various 


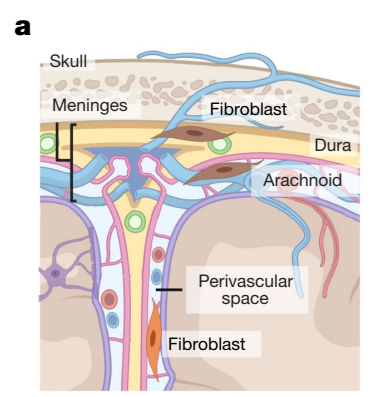

\section{b}

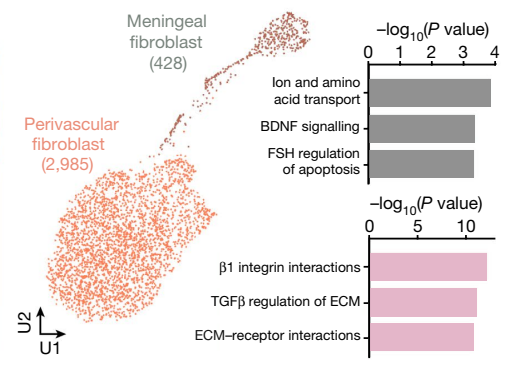

c

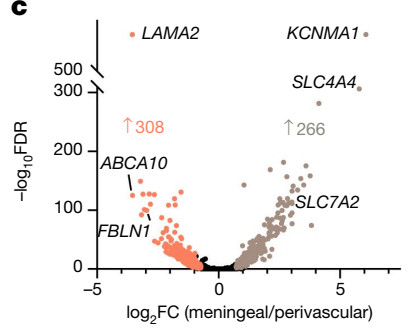

d

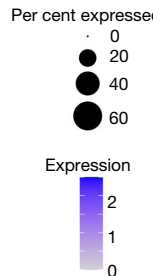

e
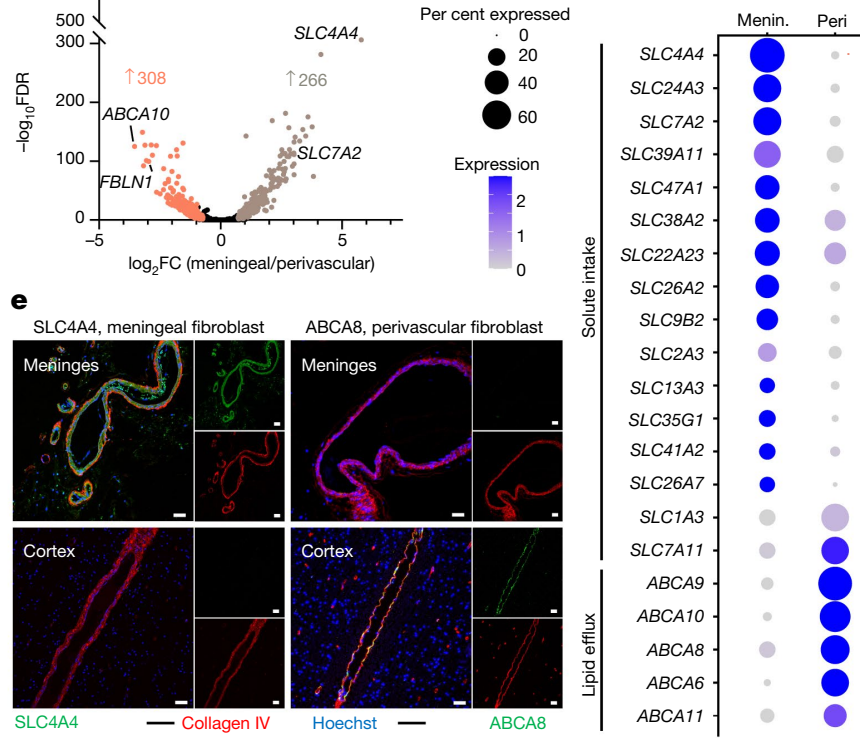

Fig. 4 | Molecular definitions for brain perivascular and meningeal fibroblasts. a, Anatomical reference of the human meninges (dura and arachnoid) and perivascular space, each with a resident fibroblast population. b, Brain fibroblast heterogeneity. UMAP of 2,985 human perivascular fibroblast-like nuclei and $\mathbf{4 2 8}$ meningeal fibroblast nuclei. Enriched biological pathways derived from respective fibroblast cell-type markers (Supplementary Table 2; $P<0.05$, cumulative hypergeometric test).c, DEGs between perivascular (left, orange) and meningeal (right, brown) fibroblasts (MAST, Benjamini-Hochberg correction; false discovery rate (FDR) $<0.01$ and $\log \mathrm{FC}>0.5\left(\log _{2} \mathrm{FC}>0.72\right)$ to be coloured significant). $\mathrm{d}$, Expression of all differentially expressed (from c) SLC and ABC family members across perivascular (peri.) and meningeal (menin.) fibroblasts. e, Immunostaining validation of polarized meningeal and perivascular fibroblast transporter expression: the meningeal fibroblast-specific influx pump SLC4A4 (left, green) stains selectively in the meninges but not in the cortical vasculature (red); and vice versa for the perivascular-specific efflux pump ABCA8 (right, green). Scale bars, $50 \mu \mathrm{m}$.

cell types, like microglia ${ }^{23}$. We isolated BECs from 12-14-month-old Thy1-hAPP ${ }^{\mathrm{Lon}, S w e}$ mice $^{37}$ (and littermate wild-type controls) and processed them for single-cell sequencing. Of note, we observed minimal overlap between human AD and mouse hAPP BECDEGs (Fig. $5 f$ ). Finally, because AD pathology begins and spreads through a very consistent regional pattern, we assessed the effect of $A D$ on brain regional vascular specialization. We found that regional differences in cell density and transcriptional profiles were largely erased in $\mathrm{AD}$ (Extended Data Fig. 8l, m)-suggesting impairments in brain-region-specific vascular functions.

Together, these findings show that patients with AD exhibit heterogeneous cell-type-, zonation-, region- and species-specific vulnerabilities and perturbations across the brain vasculature that require single-cell approaches to profile.

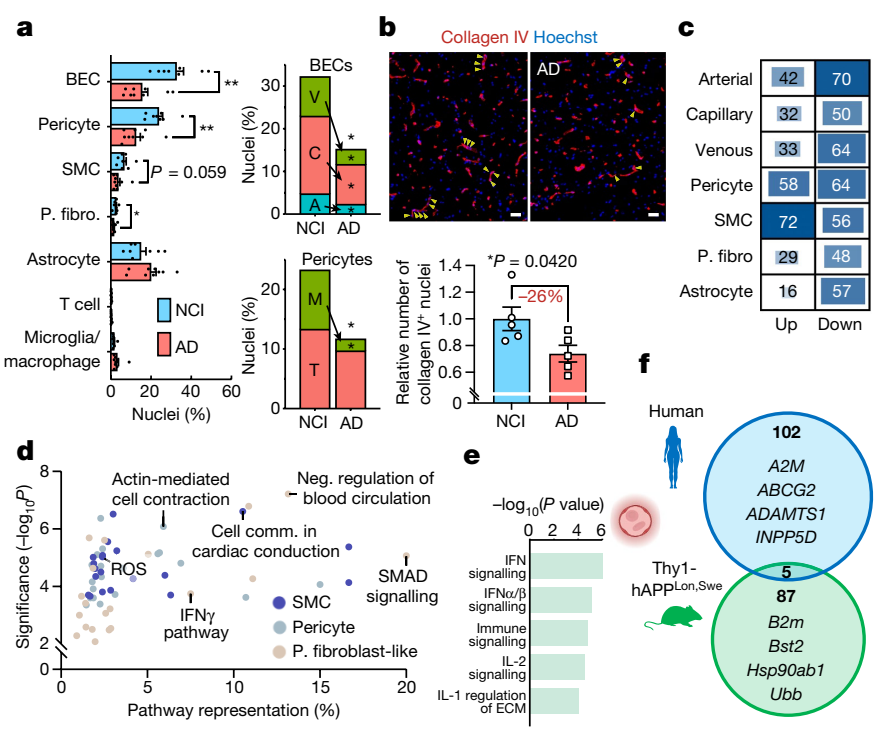

Fig. 5 | Vascular cell-type-specific perturbations in Alzheimer's disease. a, Selective vascular cell vulnerability. Left, proportion of cell types captured in individuals with $\mathrm{AD}$ and individuals with $\mathrm{NCI}$ (left). Right, proportion of $\mathrm{BEC}$ and pericyte subpopulations in $\mathrm{AD}$ and $\mathrm{NCI}$ (right) $(n=8 \mathrm{NCI}, n=9 \mathrm{AD}$; two-sided $t$-test; mean \pm s.e.m.). ${ }^{* *} P=0.002$ (BEC), ${ }^{* *} P=0.003$ (pericyte), ${ }^{*} P=0.0461$ (fibroblast) (left). ${ }^{*} P<0.05$ (right). $b$, Immunohistochemical validation of a loss of vascular cell density in $\mathrm{AD}$ (number of Hoechst ${ }^{+}$nuclei within collagen $\mathrm{IV}^{+}$vasculature). Scale bars, $50 \mu \mathrm{m}(n=5 \mathrm{NCl}$ and AD; nested two-sided $t$-test; mean \pm s.e.m.). Yellow arrows denote example vascular nuclei. c, DEG counts for each cell type in AD. The intensity of the blue colour and the size of the squares are proportional to entry values. d, Enriched biological pathways from AD DEGs in pericytes, SMCs and perivascular fibroblast-like cells, plotted by pathway representation (in a given pathway, what proportion of all members are DEGs) and significance $\left(-\log _{10} P\right)$ of pathway enrichment. ROS, reactive oxygen species. e, Enriched biological pathways from genes upregulated in individuals with AD who are carriers of $A P O E 4$, in capillary and venous endothelial cells $(P<0.05$, cumulative hypergeometric test).f, Venn diagram comparing BEC DEGs in samples from individuals with AD compared to those from the Thyl-hAPP T41B ${ }^{\text {Lon,Swe }}$ amyloidosis mouse mode ${ }^{37}$. Only genes with human-mouse orthologues are shown, and the absolute logFC threshold for calling DEGs in mouse APP BECs was lowered to 0.15 (by half) to ensure that claims of limited overlap with human BECs were robust.

\section{AD GWAS variants in human BBB cell types}

A major goal of biomedical research is to understand how genetic variation contributes to disease. GWASs have nominated genes that contribute to AD risk (hereafter referred to as AD GWAS genes). Previous studies strongly implicate microglia as the major cell type that expresses AD GWAS genes ${ }^{23,38-41}$. We wondered, however, whether the unintended depletion of brain vascular cells prematurely dismissed their potential contributions. We curated AD GWASs ${ }^{39-41}$ to order the top 45 risk genes. With our expanded survey of brain cell types, we calculated the cell-type proportional expression for each GWAS gene using expression weighted cell type enrichment (EWCE ${ }^{42}$. We indeed observed among brain parenchymal cells a specific microglial signature for top AD GWAS genes such as TREM2, MS4A6A, CR1 and SPI1 that are now under intense mechanistic study (Fig. 6a, right).

We noticed that several GWAS genes were strongly expressed in human brain vascular and perivascular cell types (Fig. 6a, left, Extended Data Fig. 9). These included two genes previously implicated in the mouse vasculature, PICALM and $C D 2 A P^{43,44}$. But they also included surprising genes, such as the immune-related PLCG2 and $H L A-D R B 1$ or HLA-DRB5 in arterial cells, endocytic INPPSD and USP6NL in capillaries, and ECM-related ADAMTS1, ADAMTS4, FERMT2 and AGRN in SMCs and 


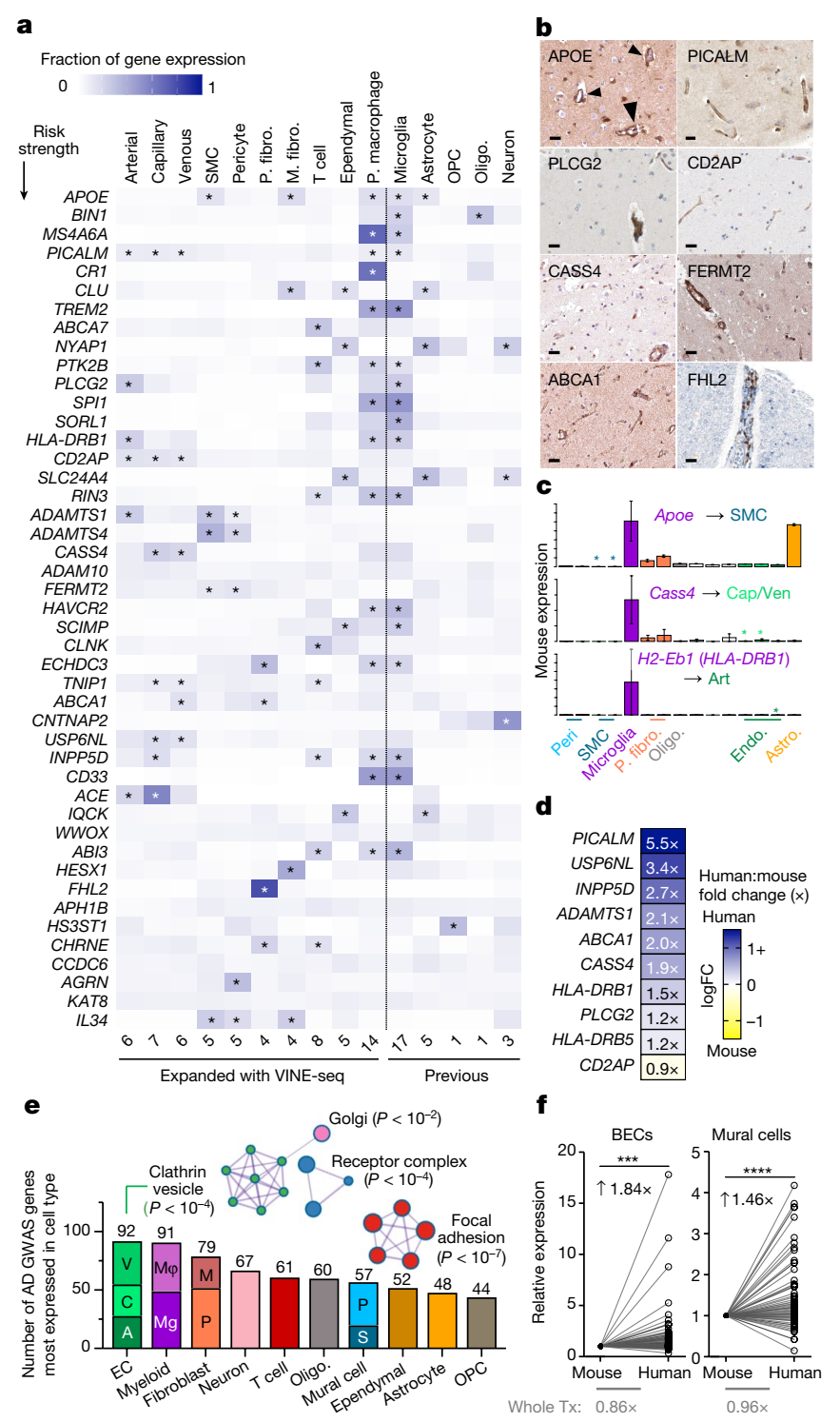

Fig. 6 | GWAS disease variants are enriched in the human brain vasculature. a, Proportional expression of the top 45 AD GWAS genes across all major brain cell types. Expression values for a given gene sums to 1 across cell types using the EWCE method ${ }^{42}$. Genes ordered in approximate risk strength ${ }^{39-41,51}$. Asterisks denote the strongest expressing cell types. Cells to the left of the dashed line are from the vasculature, newly added here; to the right, parenchymal cells captured before. Numbers on the bottom summarize the number of GWAS genes enriched in a given cell type. $M S 4 A 6 A$ represents the average expression of $M S 4 A 6 A$, $M S 4 A 4 A$ and $M S 4 A 4 E$; likewise, $H L A-D R B 1$ averages $H L A-D R B 1$ and $H L A-D R B 5$. EPHA1 was not robustly detected. b, Immunohistochemical confirmation of the vascular localization of proteins encoded by top AD GWAS genes from a. Scale bars, $25 \mu \mathrm{m}$. Arrowheads in APOE point to signal around larger-diameter vessels, consistent with SMC expression. Image credit: Human Protein Atlas ${ }^{25}$ (http:// www.proteinatlas.org).c, Examples of genes expressed specifically in mouse microglia but then also expressed in human brain vascular cell types ( $n$ of around
3,500 whole-cell mouse transcriptomes ${ }^{12}$, mean value \pm s.e.m.). Asterisk denotes human cell types that express the AD riskgene. d, BEC heat map of top AD GWAS genes coloured by logFC(human/mouse) and labelled by the linear fold change (human/mouse) value.e, Quantification of the number of AD and AD-related trait GWAS genes ${ }^{17}$ most expressed in a given cell type. 383 of 650 genes $(59 \%)$ mapped to vascular or perivascular cell types. Protein-protein interaction (PPI) network of gene ontology $(\mathrm{GO})$ cellular components $(P<0.05$, cumulative hypergeometric test). $\mathrm{EC}$, endothelial cells ( $\mathrm{A}$, arterial; $\mathrm{C}$, capillary; $\mathrm{V}$, venous). In myeloid cells, $\mathrm{Mg}$, microglia; $\mathrm{M} \varphi$, macrophage. In fibroblasts, $\mathrm{M}$, meningeal; $\mathrm{P}$, perivascular. In mural cells, S, SMC; P, pericyte. OPC, oligodendrocyte precursor cell.f, Human enrichment of the AD-related trait GWASgenes ${ }^{17}$ that are most highly expressed in BECs (left) and in mural cells (right). In contrast to GWAS genes, the ratio of human to mouse expression across the overall transcriptome (Tx) is less than or around 1 for both cell types (bottom, paired two-sided $t$-test, ${ }^{* * * *} P<0.0001$ and $^{* * *} P=0.0002$ ). pericytes (Fig. 6a). Within pericytes, expression varied across $\mathrm{M}$ - and T-pericyte subtypes (Extended Data Fig. 9a). $A P O E$, linked to myeloid cells and astrocytes, was robustly expressed in human SMCs and meningeal fibroblasts. Several GWAS genes such as $A B C A 7$ and $C L N K$ enrich in $T$ cells, and independent datasets show minimal expression in other brain cell types (Extended Data Fig. 9b). Several genes such as $A B C A 1$, $F H L 2, H E S X 1$ and IL34 enrich in fibroblasts. Notably, we confirmed our findings by immunostaining of proteins encoded by vascular GWAS genes, such as CASS4, FERMT2, PLCG2 and FHL2 (Fig. 6b, Extended Data Fig. 9c). Most GWAS genes were expressed similarly between the hippocampus and cortex (Extended Data Fig. 9d). In total, at least 30 of the top $45 \mathrm{AD}$ GWAS genes are enriched in cells of the human brain vasculature (not including those solely in perivascular macrophages), suggesting thorough vascular and perivascular involvement in $\mathrm{AD}$.

We next wondered whether these genes are expressed in different cell types between mice and humans. Many genes, like APOE, CASS4, 
INPP5D and $H L A-D R B 1$, were predominately microglial in mice ${ }^{12}$ but then also exhibited vascular expression in humans (Fig. 6c, Extended Data Fig. 9e). Nearly every top AD GWAS gene expressed in BECs showed greater expression in humans (Fig. 6d). Together, these data suggest a partial evolutionary transfer of AD risk genes and pathways from microglia to the vasculature from mice to humans (Extended Data Fig. 9f).

We broadened our scope to hundreds of GWAS genes for AD and AD-related traits ${ }^{17}$. We observed robust, cell-type-specific vascular and perivascular expression (Extended Data Fig.10). For each gene, we assigned the cell type with the strongest expression, and discovered that BECs contained the most AD-related GWAS genes, followed by microglia or macrophages (Fig. 6e, Supplementary Table 8). Within BECs, AD-related GWAS genes enriched in protein endo- and transcytosis components, such as receptor and clathrin vesicle components (Fig. 6d, e). A decline in BEC clathrin-mediated transcytosis with age has recently been demonstrated ${ }^{8}$ with age, suggesting one mechanism by which ageing and risk genes converge to increase AD risk. In total, over half of AD-related GWAS genes mapped to vascular or perivascular cell types (383 of 651). As with top GWAS genes, we observed enhanced human over mouse expression of AD-related genes in BECs and pericytes (Fig. 6f). Of note, this human-enhanced expression is not observed for the whole transcriptome.

Together, these data provide a more comprehensive understanding of the diverse cell types that contribute to AD risk. We suggest that an evolutionarily expanded vascular-microglia axis underlies the genetic risk for $\mathrm{AD}$ through shared protein clearance (BEC-microglia) and inflammatory pathways (BEC-T cell-microglia) (Fig. 6e, Extended Data Fig. 9f).

\section{Discussion}

We report here 143,793 single-cell, genome-wide quantitative transcriptomes from the human brain vasculature in health and AD. We molecularly define the principal vascular cell types; their differences by brain region and species; and the organizational principles of endothelial, mural and fibroblast-like cells. We reveal selective vulnerability of vascular subpopulations and transcriptomic perturbations associated with clinically diagnosed AD; and the expression of AD GWAS genes across human brain vascular cell types, confirmed in situ. Data are available to browse at https://twc-stanford.shinyapps. io/human_bbb.

AD has been linked to $\beta$-amyloid metabolism, cholesterol and lipid metabolism, innate immunity and endocytosis pathways operating in neurons and glia ${ }^{43}$. We expand the cell types involved; for example, debris clearance through BEC clathrin-mediated endocytosis, and adaptive $T$ cells in addition to innate immunity. We propose that the expansion of the human brain, brain activity and activity by-products (like $\beta$-amyloid ${ }^{45}$ ) necessitate enhanced clearance mechanisms and neuroimmune surveillance. In this model, microglia are still frontline participants in AD pathogenesis. But more than in mice, human vascular and perivascular cells take part. For example, microglial clearance functions can become overwhelmed ${ }^{46}$, diverting debris to BECs. Indeed, microglial depletion results in cerebral amyloid angiopathy ${ }^{47}$. But unlike microglia, vascular cells do not proliferate efficiently. Thus, constant vascular exposure to debris like $\beta$-amyloid triggers cell death, dysfunction and impaired blood and CSF flow. Previous work identified a population of $C D 8 \mathrm{~T}_{\mathrm{EMRA}}$ cells that is clonally expanded in $\mathrm{AD} \mathrm{CSF}^{48}$, potentially influenced by T cell GWAS genes. Together, we suggest an intertwined microglia-vascular axis expanded in humans, with vascular cells having an auxiliary role through shared endocytosis and inflammatory pathways. We note though the likelihood of additional human brain vascular contributions, as evidenced by SMC-, pericyte- and fibroblast-enriched GWAS genes of unclear function. In general, disease risk variants enrich in gene-expression-regulating enhancer regions ${ }^{49}$ that undergo accelerated species divergence ${ }^{50}$, providing one potential explanation for human-specific expression and dysregulation of AD risk genes in cerebrovascular cell types.

The field now has a near-complete census of human brain cell types. This atlas markedly expands the number of human brain vascular cell type and zonation markers to inform research, such as identifying vessels, validating organoid fidelity or deconvoluting bulk RNA-seq datasets. Our work also facilitates translational opportunities. This dataset provides targets for antibodies and other modalities to deliver therapeutic agents to the brain. VINE-seq enables the study of brain vascular contributions to various diseases, such as stroke, multiple sclerosis and COVID-19. But as with previous snRNA-seq studies ${ }^{16-19}$, it will be important to distinguish which vascular transcriptional perturbations respond to versus drive disease, clarify their links to clinical and pathological traits, and investigate the mechanisms by which vascular-expressed AD variants confer disease risk. Overall, VINE-seq and the ensuing single-cell atlas provide a blueprint for studying the molecular basis of the human brain vasculature.

\section{Online content}

Any methods, additional references, Nature Research reporting summaries, source data, extended data, supplementary information, acknowledgements, peer review information; details of author contributions and competing interests; and statements of data and code availability are available at https://doi.org/10.1038/s41586-021-04369-3.

1. Feigin, V. L. et al. Global and regional burden of stroke during 1990-2010: findings from the Global Burden of Disease Study 2010. Lancet 383, 245-255 (2014).

2. Chow, B. W. \& Gu, C. The molecular constituents of the blood-brain barrier. Trends Neurosci. 38, 598-608 (2015).

3. Profaci, C. P., Munji, R. N., Pulido, R. S. \& Daneman, R. The blood-brain barrier in health and disease: important unanswered questions. J. Exp. Med. 217, e20190062 (2020).

4. Obermeier, B., Daneman, R. \& Ransohoff, R. M. Development, maintenance and disruption of the blood-brain barrier. Nat. Med. 19, 1584-1596 (2013).

5. Sweeney, M. D., Zhao, Z., Montagne, A., Nelson, A. R. \& Zlokovic, B. V. Blood-brain barrier: from physiology to disease and back. Physiol. Rev. 99, 21-78 (2019).

6. Iadecola, C. The pathobiology of vascular dementia. Neuron 80, 844-866 (2013).

7. Pardridge, W. M. Drug transport across the blood-brain barrier. J. Cereb. Blood Flow Metab. 32, 1959-1972 (2012).

8. Yang, A. C. et al. Physiological blood-brain transport is impaired with age by a shift in transcytosis. Nature 583, 425-430 (2020).

9. Daneman, R., Zhou, L., Kebede, A. A. \& Barres, B. A. Pericytes are required for blood-brain barrier integrity during embryogenesis. Nature 468, 562-566 (2010).

10. Armulik, A. et al. Pericytes regulate the blood-brain barrier. Nature 468, 557-561 (2010).

11. Janzer, R. C. \& Raff, M. C. Astrocytes induce blood-brain barrier properties in endothelial cells. Nature 325, 253-257 (1987).

12. Vanlandewijck, M. et al. A molecular atlas of cell types and zonation in the brain vasculature. Nature 554, 475-480 (2018).

13. Sabbagh, M. F. et al. Transcriptional and epigenomic landscapes of CNS and non-CNS vascular endothelial cells. Elife 7, e36187 (2018).

14. Kalucka, J. et al. Single-cell transcriptome atlas of murine endothelial cells. Cell 180, 764-779 (2020)

15. Chen, M. B. et al. Brain endothelial cells are exquisite sensors of age-related circulatory cues. Cell Rep. 30, 4418-4432 (2020).

16. Mathys, H. et al. Single-cell transcriptomic analysis of Alzheimer's disease. Nature $\mathbf{5 7 0}$, 332-337 (2019).

17. Grubman, A. et al. A single-cell atlas of entorhinal cortex from individuals with Alzheimer's disease reveals cell-type-specific gene expression regulation. Nat. Neurosci. 22, 2087-2097 (2019).

18. Jäkel, S. et al. Altered human oligodendrocyte heterogeneity in multiple sclerosis. Nature 566, 543-547 (2019).

19. Velmeshev, D. et al. Single-cell genomics identifies cell type-specific molecular changes in autism. Science 364, 685-689 (2019).

20. Keller, D., Erö, C. \& Markram, H. Cell densities in the mouse brain: a systematic review. Front. Neuroanat. 12, 83 (2018).

21. Niedowicz, D. M. et al. Obesity and diabetes cause cognitive dysfunction in the absence of accelerated $\beta$-amyloid deposition in a novel murine model of mixed or vascular dementia. Acta Neuropathol. Commun. 2, 64 (2014).

22. Montagne, A. et al. Blood-brain barrier breakdown in the aging human hippocampus. Neuron 85, 296-302 (2015)

23. Geirsdottir, L. et al. Cross-species single-cell analysis reveals divergence of the primate microglia program. Cell 179, 1609-1622 (2019).

24. Trapnell, C. et al. The dynamics and regulators of cell fate decisions are revealed by pseudotemporal ordering of single cells. Nat. Biotechnol. 32, 381-386 (2014).

25. Uhlén, M. et al. Tissue-based map of the human proteome. Science 347, 1260419 (2015).

26. De Meyer, S. F., Stoll, G., Wagner, D. D. \& Kleinschnitz, C. Von Willebrand factor: an emerging target in stroke therapy. Stroke 43, 599-606 (2012). 


\section{Article}

27. Mao, M., Alavi, M. V., Labelle-Dumais, C. \& Gould, D. B. Type IV collagens and basement membrane diseases: cell biology and pathogenic mechanisms. Curr. Top. Membr. 76, 61-116 (2015).

28. DeSisto, J. et al. Single-cell transcriptomic analyses of the developing meninges reveal meningeal fibroblast diversity and function. Dev. Cell 54, 43-59 (2020).

29. Louveau, A. et al. Structural and functional features of central nervous system lymphatic vessels. Nature 523, 337-341 (2015).

30. Aspelund, A. et al. A dural lymphatic vascular system that drains brain interstitial fluid and macromolecules. J. Exp. Med. 212, 991-999. (2015).

31. Dorrier, C. E. et al. CNS fibroblasts form a fibrotic scar in response to immune cell infiltration. Nat. Neurosci. 24, 234-244 (2021).

32. Iliff, J. J. et al. A paravascular pathway facilitates CSF flow through the brain parenchyma and the clearance of interstitial solutes, including amyloid $\beta$. Sci. Transl. Med. 4, 147ra111 (2012).

33. Keren-Shaul, $\mathrm{H}$. et al. A unique microglia type associated with restricting development of Alzheimer's disease. Cell 169, 1276-1290 (2017).

34. Brown, W. R. A review of string vessels or collapsed, empty basement membrane tubes. J. Alzheimer's Dis. 21, 725-739 (2010)

35. Roher, A. E. et al. Cerebral blood flow in Alzheimer's disease. Vasc. Health Risk Manag. 8, 599 (2012).

36. Montagne, A. et al. APOE4 leads to blood-brain barrier dysfunction predicting cognitive decline. Nature 581, 71-76 (2020)

37. Rockenstein, E., Mallory, M., Mante, M., Sisk, A. \& Masliaha, E. Early formation of mature amyloid- $\beta$ protein deposits in a mutant APP transgenic model depends on levels of $A \beta_{1-42}$ J. Neurosci. Res. 66, 573-582 (2001).

38. Nott, A. et al. Brain cell type-specific enhancer-promoter interactome maps and disease-risk association. Science 366, 1134-1139 (2019).

39. Lambert, J. C. et al. Meta-analysis of 74,046 individuals identifies 11 new susceptibility loci for Alzheimer's disease. Nat. Genet. 45, 1452-1458 (2013).

40. Kunkle, B. W. et al. Genetic meta-analysis of diagnosed Alzheimer's disease identifies new risk loci and implicates $A \beta$, tau, immunity and lipid processing. Nat. Genet. 51, 414-430 (2019).
41. Jansen, I. E. et al. Genome-wide meta-analysis identifies new loci and functional pathways influencing Alzheimer's disease risk. Nat. Genet. 51, 404-413 (2019).

42. Skene, N. G. \& Grant, S. G. N. Identification of vulnerable cell types in major brain disorders using single cell transcriptomes and expression weighted cell type enrichment. Front. Neurosci. 10, 16 (2016).

43. Karch, C. M. \& Goate, A. M. Alzheimer's disease risk genes and mechanisms of disease pathogenesis. Biol. Psychiatry 77, 43-51 (2015).

44. Zhao, Z. et al. Central role for PICALM in amyloid- $\beta$ blood-brain barrier transcytosis and clearance. Nat. Neurosci. 18, 978-987 (2015)

45. Cirrito, J. R. et al. Synaptic activity regulates interstitial fluid amyloid- $\beta$ levels in vivo. Neuron 48, 913-922 (2005).

46. Safaiyan, S. et al. Age-related myelin degradation burdens the clearance function of microglia during aging. Nat. Neurosci. 19, 995-998 (2016)

47. Spangenberg, E. et al. Sustained microglial depletion with CSF1R inhibitor impairs parenchymal plaque development in an Alzheimer's disease model. Nat. Commun. 10 3758 (2019).

48. Gate, D. et al. Clonally expanded CD8 T cells patrol the cerebrospinal fluid in Alzheimer's disease. Nature 577, 399-404 (2020).

49. Farh, K. K. H. et al. Genetic and epigenetic fine mapping of causal autoimmune disease variants. Nature 518, 337-343 (2015)

50. Villar, D. et al. Enhancer evolution across 20 mammalian species. Cell 160, 554-566 (2015).

51. Wightman, D. P. et al. Largest GWAS $(N=1,126,563)$ of Alzheimer's disease implicates microglia and immune cells. Preprint at https://doi.org/10.1101/2020.11.20.20235275 (2020).

Publisher's note Springer Nature remains neutral with regard to jurisdictional claims in published maps and institutional affiliations.

(c) The Author(s), under exclusive licence to Springer Nature Limited 2022 


\section{Methods}

Isolation of vascular nuclei from frozen post-mortem brain tissue Post-mortem fresh-frozen hippocampus and superior frontal cortex tissue were obtained from the Stanford/VA/NIA Aging Clinical Research Center (ACRC) with approval from local ethics committees and patient consent. Group characteristics are presented in Supplementary Table 1 . Individuals were grouped by clinical diagnosis, with two of the individuals with $\mathrm{NCl}$ exhibiting $\beta$-amyloid plaque staining in the hippocampus, although not to a sufficient degree for an expert pathologist to diagnose AD by histopathological criteria. Clinical instead of pathological diagnosis was chosen because of potentially vascular contributions to AD independent of the well-known hallmarks of $\mathrm{AD}, \beta$-amyloid and tau pathophysiology ${ }^{6}$. All procedures were carried out on ice in a $4{ }^{\circ} \mathrm{C}$ cold room as rapidly as possible. Brain tissue ( 0.3 grams or more) was thawed on ice for $5 \mathrm{~min}$ with $5 \mathrm{ml}$ of nuclei

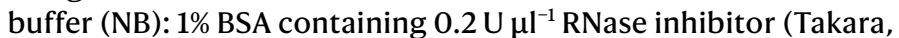
2313A) and EDTA-free protease inhibitor cocktail (Roche, 11873580001). Tissue was quickly minced and homogenized with 7-ml glass douncers (357424, Wheaton) until no visible chunks of debris remained. Similar to before ${ }^{52}$, homogenates were transferred into 50 -ml tubes containing $35 \mathrm{ml}$ of chilled 32\% dextran (D8821, Sigma) in HBSS. Samples were vigorously mixed before centrifugation at $4,400 \mathrm{~g}$ for $20 \mathrm{~min}$ with no brake. After centrifugation, samples separate into a top myelin layer, middle parenchymal layer and vascular-enriched pellet. The myelin layer was aspirated, tips were changed and the parenchymal layer was carefully removed without disturbing the pellet. Pellets were resuspended in $8 \mathrm{ml}$ of $32 \%$ dextran, transferred to $15-\mathrm{ml}$ falcon tubes, and centrifuged again. Vascular-enriched pellets were gently resuspended in $1 \mathrm{ml}$ of NB and added to pre-wetted $40-\mu \mathrm{m}$ strainers sitting on top of $50-\mathrm{ml}$ falcon tubes. From here diverging from prior protocol, strainers were washed with $10 \mathrm{ml}$ of cold $0.32 \mathrm{M}$ sucrose in PBS and $90 \mathrm{ml}$ PBS until flow through the strainers was unimpeded to deplete contaminating parenchymal cells from trapped microvessels. At this step, retained microvessels turn white in colour, indicating the removal of circulating blood cells. Strainers were switched to new collection 50-ml falcon tubes. Various techniques were tested and optimized to extract vascular cells from the isolated microvessels (for example, enzymatic digestion, TisssueRuptor, sonication and so on), but nearly all resulted in loss of nuclei integrity or low nuclei complexity (fewer than 50 median genes per nucleus). Eventually, adapting a method for the isolation of mouse splenocytes proved successful: vascular fragments were mashed four times through the cell strainer using the plunger end of a $3-\mathrm{ml}$ syringe, with intermittent elution via $10 \mathrm{ml}$ of $0.32 \mathrm{M}$ sucrose and $40 \mathrm{ml}$ of PBS. Liberated vascular cells were pelleted at $500 \mathrm{~g}$ for $10 \mathrm{~min}$ and resuspended in $1.5 \mathrm{ml}$ of EZ Prep Lysis

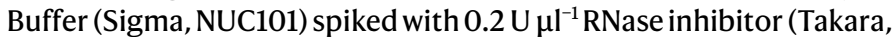
2313A) and EDTA-free protease inhibitor cocktail (Roche, 11873580001). Nuclei were homogenized with 2-ml glass douncers (D8938, Sigma) 20 times with pestle B (pestle A optional). Spiked EZ lysis buffer was added to samples up to $4 \mathrm{ml}$ and incubated on ice for $5 \mathrm{~min}$ before pelleting at $500 \mathrm{~g}$ for $6 \mathrm{~min}$. This incubation step was repeated. Debris was depleted via a sucrose gradient before flow cytometry isolation of nuclei. In brief, pelleted nuclei were resuspended in $0.5 \mathrm{ml}$ of NB before the addition of $0.9 \mathrm{ml}$ of $2.2 \mathrm{M}$ sucrose in PBS. This mixture was layered on top of $0.5 \mathrm{ml}$ of $2.2 \mathrm{M}$ sucrose and samples were centrifuged at $14,000 \mathrm{~g}$ for $45 \mathrm{~min}$ at $4{ }^{\circ} \mathrm{C}$, with no brake. Pellets were aspirated in $1 \mathrm{ml}$ of NB, filtered through a $40-\mu \mathrm{m}$ strainer (Flowmi), transferred to FACS tubes, stained with Hoechst 3342 (1:2,000, Thermo Fisher Scientific) and rabbit monoclonal anti-NeuN Alexa Fluor 647 (1:500, Abcam, ab190565), and nuclei collected on a SH800S Cell Sorter into chilled tubes containing $1 \mathrm{ml}$ of NB without protease inhibitor. In pilot runs, we noticed that the cytometer overestimated nuclei counts by around 3.4 times, and thus we sorted around 34,000 nuclei to target around 10,000 nuclei per sample. Sorted samples were inspected for lack of debris on a bright-field microscope. We note that an iodixanol gradient ${ }^{53}$ can substitute for the $2.2 \mathrm{M}$ sucrose, but that unfortunately with either gradient, flow sorting is required-unlike parenchymal myelin debris, vascular debris is not sufficiently removed by gradient centrifugation alone. Vascular debris will confound downstream cDNA traces with higher background and low molecular weight peaks.

\section{Droplet-based snRNA-seq}

For droplet-based snRNA-seq, libraries were prepared using the Chromium Single Cell 3'v3 according to the manufacturer's protocol (10x Genomics), targeting 10,000 nuclei per sample after flow sorting (Sony SH800S Cell Sorter). Fifteen PCR cycles were applied to generate cDNA before 16 cycles for final library generation. Generated snRNA-seq libraries were sequenced on S4 lanes of a NovaSeq 6000 (150 cycles, Novogene).

\section{SnRNA-seq quality control}

Gene counts were obtained by aligning reads to the hg38 genome (refdata-gex-GRCh38-2020-A) using CellRanger software (v.4.0.0) (10x Genomics). To account for unspliced nuclear transcripts, reads mapping to pre-mRNA were counted. As previously published, a cut-off value of 200 unique molecular identifiers (UMIs) was used to select single nuclei for further analysis ${ }^{16,54}$. As initial reference, the entire dataset was projected onto two-dimensional space using UMAP on the top 30 principal components ${ }^{55}$. Three approaches were combined for strict quality control: (1) outliers with a high ratio of mitochondrial (more than $5 \%$, fewer than 200 features) relative to endogenous RNAs and homotypic doublets (more than 5,000 features) were removed in Seurat ${ }^{56}$; (2) after scTransform normalization and integration, doublets and multiplets were filtered out using DoubletFinder ${ }^{57}$; and (3) after DoubletFinder, nuclei were manually inspected using known cell-type-specific marker genes, with nuclei expressing more than one cell-type-specific marker further filtered ${ }^{16,18,57-61}$. For example, BEC nuclei containing any reads for the following cell type markers were subsequently filtered: MOBP, MBP, MOG, SLC38A11, LAMA2, PDGFRB, GFAP, SLC1A2 and AQP4. We note that the vascular nuclei in previous human single-cell datasets exhibit contamination with other cell-type-specific gene markers, which potentially confounds downstream analysis. After applying these filtering steps, the dataset contained 143,793 high-quality, single nuclei.

\section{Cell annotations and differential gene expression analysis}

Seurat's integration function was used to align data with default settings. Genes were projected into principal component (PC) space using the principal component analysis (RunPCA). The first 30 dimensions were used as inputs into Seurat's FindNeighbors, FindClusters (at 0.2 resolution) and RunUMAP functions. In brief, a shared-nearest-neighbour graph was constructed based on the Euclidean distance metric in PC space, and cells were clustered using the Louvain method. RunUMAP functions with default settings were used to calculate two-dimensional UMAP coordinates and search for distinct cell populations. Positive differential expression of each cluster against all other clusters (model-based analysis of single-cell transcriptomics; MAST) was used to identify marker genes for each cluster ${ }^{62}$. We annotated cell-types using previously published marker genes ${ }^{16,19,61,63}$. For BECs, zonation specificity scores for each gene were calculated separately for arterial, capillary, and venous segments as in the following example for a given gene in capillaries:

\section{Capillary specificity score}

$$
=\text { Average }\left[\log \left(\frac{\text { Capillary } \log \mathrm{CPM}}{\text { Arterial } \log \mathrm{CPM}}\right), \log \left(\frac{\text { Capillary } \log \mathrm{CPM}}{\text { Veinous } \log \mathrm{CPM}}\right)\right] \text {. }
$$

Differential gene expression of genes comparing $\mathrm{AD}, \mathrm{ApoE} 4$, and $\mathrm{NCI}$ samples-or comparing cell type subcluster markers-was done using the MAST ${ }^{62}$ algorithm, which implements a two-part hurdle model. 


\section{Article}

Seurat natural $\log$ (fold change) $>0.5$ (absolute value), adjusted $P$ value (Bonferroni correction) $<0.01$, and expression in greater than $10 \%$ of cells in both comparison groups were required to consider a gene differentially expressed for subcluster analysis and natural $\log$ (fold change) $>0.3$ (absolute value), adjusted $P$ value (Bonferroni correction) $<0.01$, and expression in greater than $10 \%$ of cells in both comparison groups for $\mathrm{AD}$ and $\mathrm{ApoE} 4$ comparisons, both more stringent than the default Seurat settings. We incorporated age, gender and batch as covariates in our model. A more lenient threshold of the above but with natural $\log$ (fold change) $>0.2$ (absolute value) was used for brain region (that is, hippocampus versus cortex). Biological pathway and gene ontology enrichment analyses were performed using Enrichr ${ }^{64}$ or Metascape $^{65}$ with the input species set to Homo sapiens ${ }^{65}$. UpSet plots were generated using identified DEGs as inputs using the R package UpSetR $^{66}$. Diagrams were created with BioRender.

\section{Monocle trajectory analysis}

Monocle was used to generate the pseudotime trajectory analysis in brain endothelial and mural cells ${ }^{24}$. Cells were clustered in Seurat and cluster markers used as input into Monocle to infer arteriovenous relationships within endothelial cells and pericytes. Specifically, UMAP embeddings and cell subclusters generated from Seurat were converted to a cell_data_set object using SeuratWrappers (v.0.2.0) and then used as input to perform trajectory graph learning and pseudotime measurement through independent component analysis (ICA) with Monocle. Cluster marker genes identified in Seurat were used to generate a pseudotime route and plotted using the 'plot_pseudotime_heatmap' function. For mural cells, variable genes were limited to those with $\log$ [average expression] $>1$ (Seurat) for even more robust cell ordering.

\section{Cell-cell communication}

Cell-cell interactions based on the expression of known ligand-receptor pairs in different cell types were inferred using CellChatDB ${ }^{67}$ (v.0.02). In brief, we followed the official workflow and loaded the normalized counts into CellChat and applied the preprocessing functions identifyOverExpressedGenes', 'identifyOverExpressedInteractions' and 'projectData' with standard parameters set. As database we selected the 'Secreted Signaling' pathways and used the pre-compiled human 'Protein-Protein-Interactions' as a priori network information. For the main analyses the core functions 'computeCommunProb', 'computeCommunProbPathway' and 'aggregateNet' were applied using standard parameters and fixed randomization seeds. Finally, to determine the senders and receivers in the network the function 'netAnalysis_signalingRole' was applied on the 'netP' data slot.

\section{Mice}

Aged C57BL/6 male mice (19 months old) were obtained from the National Institute on Aging rodent colony. Young male C57BL/6 mice (3 months old) were obtained from The Jackson Laboratory or Charles River Laboratories. Thy1-hAPP ${ }^{\mathrm{L} \text {, Swe }}$ male mice and littermate wild-type control $^{37}$ mice were raised until 12-14 months of age. All mice were kept on 12-h light-dark cycle and provided ad libitum access to food and water. All animal care and procedures complied with the Animal Welfare Act and were in accordance with institutional guidelines and approved by the V.A. Palo Alto Committee on Animal Research and the institutional administrative panel of laboratory animal care at Stanford University.

\section{Mouse wild-type and APP T41B BEC single-cell and nuclei sequencing}

Whole cell isolation from the central nervous system followed previously described methods ${ }^{68-70}$. In brief, cortices and hippocampi were microdissected, minced and digested using the Neural Dissociation Kit (Miltenyi). Suspensions were filtered through a $100-\mu \mathrm{m}$ strainer and myelin removed by centrifugation in $0.9 \mathrm{M}$ sucrose. The remaining myelin-depleted cell suspension was blocked for $10 \mathrm{~min}$ with Fc preblock (CD16/CD32, BD 553141) on ice and stained for 20 min with antibodies to distinguish BECs $\left(\mathrm{CD} 31^{+} / \mathrm{CD} 45^{-}\right)$. BECs from 12-14-month-old Thy1-hAPP ${ }^{\text {Lon,Swe }}$ mice and littermate wild-type con$\mathrm{trol}^{37}$ mice (pool of 4-6 mice per group) were sorted into PBS with $0.1 \%$ BSA. Nuclei isolation from 4-6 month-old mouse hippocampi followed protocols adapted from previous studies ${ }^{16,17,53,61,71}$. In brief, tissue was homogenized using a glass douncer in $2 \mathrm{ml}$ of ice-cold EZ PREP buffer (Sigma, N3408) and incubated on ice for $5 \mathrm{~min}$. Centrifuged nuclei

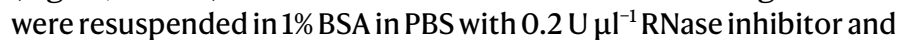
filtered through a $40-\mu \mathrm{m}$ cell strainer. Cells or nuclei were immediately counted using a Neubauer haemocytometer and loaded on a Chromium Single-Cell Instrument (10x Genomics) to generate single-cell gel-bead in emulsions (GEMs). The 10x Genomics v3 libraries were prepared as per the manufacturer's instructions. Libraries were sequenced on an Illumina NextSeq 550 (paired-end; read 1: 28 cycles; 7 index: 8 cycles, i5 index: 0 cycles; read 2: 91 cycles). De-multiplexing was performed using the Cellranger toolkit (v.3.0.0) 'cellranger mkfastq' command and the 'cellranger count' command for alignment to the mouse transcriptome, cell barcode partitioning, collapsing UMIs to transcripts, and gene-level quantification. Around 70\% sequencing saturation (more than 20,000 reads per cell) was achieved, for a median of around 2,000 genes detected per cell and around 16,500 genes detected in total. Downstream analysis using the Seurat package (v.3 $)^{72}$ was performed as previously described ${ }^{12}$, applying standard algorithms for cell filtration, feature selection and dimensionality reduction. Samples with fewer than 1,000 and more than 4,000 unique feature counts, samples with more than $15 \%$ mitochondrial RNA, samples with more than $15 \%$ small subunit ribosomal genes (Rps), and counts of more than 10,000 were excluded from the analysis. Genes were projected into PC space using the principal component analysis (RunPCA). The first 30 dimensions were used as inputs into Seurat's FindNeighbors and RunTsne functions. In brief, a shared-nearest-neighbour graph was constructed based on the Euclidean distance metric in PC space, and cells were clustered using the Louvain method. RunTsne functions with default settings was used to calculate two-dimensional $t$-distributed stochastic neighbour embedding ( $t$-SNE) coordinates and search for distinct cell populations. Cells and clusters were then visualized using three-dimensional $t$-SNE embedding on the same distance metric. Differential gene expression analysis was done by applying MAST. Significant DEGs in Thy1-hAPP ${ }^{\text {Lon,Swe }}$ BECs were called by log(fold change) $>0.15$ (absolute value), adjusted $P$ value (Bonferroni correction) $<0.01$. This lowered $\log$ (fold change) was to ensure our claims of limited overlap with human AD BECs were robust.

\section{GWAS analysis}

For calculation of proportional cell-type-specific gene expression, we followed the EWCE method described in a previous study ${ }^{42}$, and used previously on human snRNA-seq data ${ }^{17}$. For AD analysis, we compiled a list of top GWAS risk genes from ref. ${ }^{39}$, ref. $^{40}$ and ref. ${ }^{41}$, sorted descending by approximate $P$ value. The expression of each gene sums to 1 across the cell types, with each heat map cell showing the fraction of total gene expression as determined from EWCE analysis. The set of $720 \mathrm{AD}$ and AD-related trait GWAS genes were obtained from ref. ${ }^{17}$, and using EWCE analysis, the strongest expressing cell type was determined for each gene. The original list was slightly parsed to 720 , as several genes were not detected as expressed in our dataset.

\section{Immunohistochemistry}

Fresh-frozen human brain tissue from individuals with $\mathrm{NCI}$ and from individuals with $\mathrm{AD}$ (hippocampus and superior frontal cortex adjacent to tissue processed for snRNA-seq as well as meninges) was subjected to immunohistochemistry (IHC). Ten-micrometre sections mounted on SuperFrost Plus glass slides were fixed with $4 \%$ paraformaldehyde (Electron Microscopy Services, 15714S) diluted in PBS at $4{ }^{\circ} \mathrm{C}$ for $15 \mathrm{~min}$ 
before dehydration via an ethanol series or air drying. Sections were blocked in TBS++ (TBS + 3\% donkey serum (130787, Jackson ImmunoResearch) $+0.25 \%$ Triton X-100 (T8787, Sigma-Aldrich)) for $1.5 \mathrm{~h}$ at room temperature. Sections were incubated with primary antibodies at $4{ }^{\circ} \mathrm{C}$ overnight: goat polyclonal anti-collagen type IV (1:200, AB769, Sigma), rabbit polyclonal anti-CYP1B1 (1:100, HPA026863, Atlas Antibodies), rabbit polyclonal anti-SLC4A4 (1:100, HPA035628, Atlas Antibodies), rabbit polyclonal anti-SLC47A1 (1:100, HPA021987, Atlas Antibodies), rabbit polyclonal anti-ABCA8 (1:100, HPA044914, Atlas Antibodies), mouse monoclonal anti-CD31 (1:100, JC70A, Dako), rabbit polyclonal anti-VWF (1:100, GA527, Dako), rabbit polyclonal anti-SLC39A10 (1:100, HPA066087, Atlas Antibodies), rabbit polyclonal antiALPL (1:100, HPA007105, Atlas Antibodies), rabbit polyclonal anti-A2M (1:100, HPA002265, Atlas Antibodies), rabbit monoclonal anti- $\beta$-amyloid (1:500, clone D54D2 XP, CST) and mouse monoclonal anti-actin, $\alpha$-smooth muscle-Cy3 (1:100, clone 1A4, Sigma). Sections were washed, stained with Alexa Fluor-conjugated secondary antibodies (1:250) and Hoechst 33342 (1:2,000, H3570, Thermo Fisher Scientific), mounted and coverslipped with ProLong Gold (Life Technologies) or VECTASHIELD (Vector Laboratories) before imaging on a confocal laser scanning microscope (Zeiss LSM880). Age-related autofluorescence was quenched before mounting with Sudan Black B, as before ${ }^{8,70}$. National Institutes of Health ImageJ software was used to quantify the number of Hoechst $^{+}$nuclei per image, the percentage of vasculature (collagen IV), the number of Hoechst ${ }^{+}$nuclei within collagen $\mathrm{IV}^{+}$vasculature, or the predicted DEG SLC39A10 among CD31 ${ }^{+}$vasculature, following previously described protocols ${ }^{8,15,73}$. In short, at least five images were stained per patient, and imaging and analyses were performed by a blinded observer.

\section{Statistics and reproducibility}

Immunostaining validation experiments were repeated independently at least twice with similar results. As indicated in the figure legends, some immunostaining images come from the Human Protein Atlas ${ }^{25,74}$ and are available at https://www.proteinatlas.org/.

\section{Reporting summary}

Further information on research design is available in the Nature Research Reporting Summary linked to this paper.

\section{Data availability}

Raw sequencing data have been deposited in the NCBI Gene Expression Omnibus (GEO) under accession code GSE163577. Data are also available to explore via an interactive web browser: https://twc-stanford. shinyapps.io/human_bbb.

52. Lee, Y. K., Uchida, H., Smith, H., Ito, A., \& Sanchez, T. The isolation and molecular characterization of cerebral microvessels. Nat. Protoc. 14, 3059-3081 (2019).

53. Corces, M. R. et al. An improved ATAC-seq protocol reduces background and enables interrogation of frozen tissues. Nat. Methods 14, 959-962 (2017).

54. Yang, A. C. et al. Dysregulation of brain and choroid plexus cell types in severe COVID-19. Nature 595, 565-571 (2021).

55. McInnes, L., Healy, J. \& Melville, J. UMAP: uniform manifold approximation and projection for dimension reduction. Preprint at https://arxiv.org/abs/1802.03426 (2018).

56. Satija, R., Farrell, J. A., Gennert, D., Schier, A. F., \& Regev, A. Spatial reconstruction of single-cell gene expression data. Nat. Biotechnol. 33, 495-502 (2015).

57. McGinnis, C. S., Murrow, L. M., \& Gartner, Z. J. DoubletFinder: doublet detection in single-cell RNA sequencing data using artificial nearest neighbors. Cell Syst. 8, 329-337 (2019).

58. Zeisel, A. et al. Molecular architecture of the mouse nervous system. Cell 174, 999-1014 (2018).

59. Zeisel, A. et al. Cell types in the mouse cortex and hippocampus revealed by single-cell RNA-seq. Science 347, 1138-1142 (2015).

60. Yang, A. C. et al. Dysregulation of brain and choroid plexus cell types in severe COVID-19. Nature 595, 565-571 (2021).
61. Zhou, Y. et al. Human and mouse single-nucleus transcriptomics reveal TREM2-dependent and TREM2-independent cellular responses in Alzheimer's disease. Nat. Med. 26, 131-142 (2020).

62. Finak, G. et al. MAST: a flexible statistical framework for assessing transcriptional changes and characterizing heterogeneity in single-cell RNA sequencing data. Genome Biol. 16, 278 (2015).

63. Lake, B. B. et al. Integrative single-cell analysis of transcriptional and epigenetic states in the human adult brain. Nat. Biotechnol. 36, 70-80 (2018).

64. Chen, E. Y. et al. Enrichr: interactive and collaborative HTML5 gene list enrichment analysis tool. BMC Bioinformatics 14, 128 (2013).

65. Zhou, Y. et al. Metascape provides a biologist-oriented resource for the analysis of systems-level datasets. Nat. Commun. 10, 1523 (2019).

66. Conway, J. R., Lex, A. \& Gehlenborg, N. UpSetR: an R package for the visualization of intersecting sets and their properties. Bioinformatics 33, 2938-2940 (2017).

67. Jin, S. et al. Inference and analysis of cell-cell communication using CellChat. Nat. Commun. 12, 1088 (2021).

68. The Tabula Muris Consortium. Single-cell transcriptomics of 20 mouse organs creates a Tabula Muris. Nature 562, 367-372 (2018).

69. Zuchero, Y. J. Y. et al. Discovery of novel blood-brain barrier targets to enhance brain uptake of therapeutic antibodies. Neuron 89, 70-82 (2016).

70. Yousef, H., et al. Aged blood impairs hippocampal neural precursor activity and activates microglia via brain endothelial cell VCAM1. Nat. Med. 25, 988-1000 (2019).

71. Swiech, L. et al. In vivo interrogation of gene function in the mammalian brain using CRISPR-Cas9. Nat. Biotechnol. 33, 102-106 (2015).

72. Butler, A., Hoffman, P., Smibert, P., Papalexi, E. \& Satija, R. Integrating single-cell transcriptomic data across different conditions, technologies, and species. Nat. Biotechnol. 36, 411-420 (2018).

73. Yang, A. C. et al. Multiple click-selective tRNA Synthetases expand mammalian cell-specific proteomics. J. Am. Chem. Soc. 140, 7046-7051 (2018).

74. Thul, P. J. et al. A subcellular map of the human proteome. Science 356, eaal3321 (2017)

75. Butovsky, O. et al. Identification of a unique TGF- $\beta$-dependent molecular and functional signature in microglia. Nat. Neurosci. 17, 131-143 (2014).

76. Szabo, P. A. et al. Single-cell transcriptomics of human T cells reveals tissue and activation signatures in health and disease. Nat. Commun. 10, 4706 (2019).

77. Iadecola, C., Anrather, J. \& Kamel, H. Effects of COVID-19 on the nervous system. Cell 183, 16-27 (2020).

78. Månberg, A. et al. Altered perivascular fibroblast activity precedes ALS disease onset. Nat. Med. 27, 640-646 (2021).

79. Parker, K. R. et al. Single-cell analyses identify brain mural cells expressing CD19 as potential off-tumor targets for CAR-T immunotherapies. Cell 183, 126-142 (2020).

80. Vanlandewijck, M. et al. A molecular atlas of cell types and zonation in the brain vasculature. Nature 554, 475-480 (2018).

81. Zhang, Y. et al. Purification and characterization of progenitor and mature human astrocytes reveals transcriptional and functional differences with mouse. Neuron $\mathbf{8 9}$, 37-53 (2016).

Acknowledgements We thank T. Iram, E. Tapp, N. Lu, M. Haney, O. Hahn, M. J. Estrada, S. M. Shi and other members of the Wyss-Coray laboratory for feedback and support; H. Mathys, D. A. Bennett and participants in the CSHL BBB 2021 meeting for advice; and H. Zhang and K. Dickey for laboratory management. This work was funded by the NOMIS Foundation (T.W.-C.), the National Institute on Aging (T32-AG0047126 to A.C.Y. and 1RF1AG059694 to T.W.-C), Nan Fung Life Sciences (T.W.-C.), the Bertarelli Brain Rejuvenation Sequencing Cluster (an initiative of the Stanford Wu Tsai Neurosciences Institute) and the Stanford Alzheimer's Disease Research Center (P30 AG066515). This work was supported by a grant from the Simons Foundation Award (811253TWC). A.C.Y. was supported by a Siebel Scholarship. F.K. and A.K. are part of the CORSAAR study supported by the State of Saarland, the Saarland University and the Rolf M. Schwiete Stiftung. This study was supported by the AHA-Allen Initiative in Brain Health and Cognitive Impairment (19PABHI34580007). The statements in this work are solely the responsibility of the authors and do not necessarily represent the views of the American Heart Association (AHA) or the Paul G. Allen Frontiers Group. Graphics were created with BioRender. com.

Author contributions A.C.Y. and T.W.-C. conceptualized the study. A.C.Y. devised the isolation method. M.W.M. and W.J.S.-S. provided and A.C.Y. organized tissue samples. D.P.L. and A.C.Y. performed tissue dissociations. N.S., R.T.V., D.G., K.C., H.S. and A.C.Y. prepared libraries for sequencing. R.T.V., F.K., A.K., C.A.M., M.B.C., R.P., A.S., N.K., J.A.S. and A.C.Y. performed computational analysis. D.P.L., C.A.M., M.A., D.G., E.Y.W., J.L. A.T., P.C. and A.C.Y. performed immunohistochemical stains. P.M.L. developed the searchable web interface (Shiny app). C.A.M. and A.C.Y. drew diagrams. A.C.Y. wrote the manuscript with input from all authors. A.C.Y. and T.W.-C. supervised the study.

Competing interests T.W.-C. is a co-founder and scientific advisor of Alkahest. A.C.Y., R.T.V. and T.W.-C. are co-founders and scientific advisors of Qinotto.

\section{Additional information}

Supplementary information The online version contains supplementary material available at https://doi.org/10.1038/s41586-021-04369-3.

Correspondence and requests for materials should be addressed to Andrew C. Yang or Tony Wyss-Coray.

Peer review information Nature thanks Trygve Bakken, Neelroop Parikshak and the other, anonymous, reviewer(s) for their contribution to the peer review of this work. Reprints and permissions information is available at http://www.nature.com/reprints. 
a VINE-seq method for human brain vascular nuclei

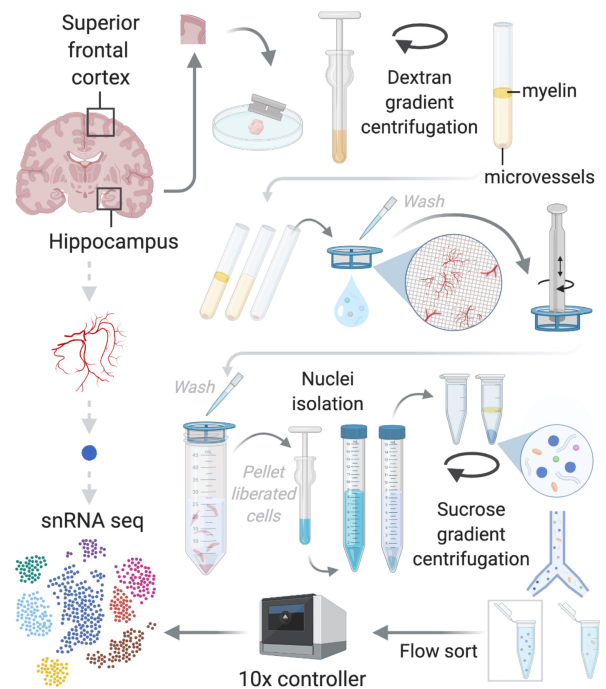

d Vascular cell type numbers captured
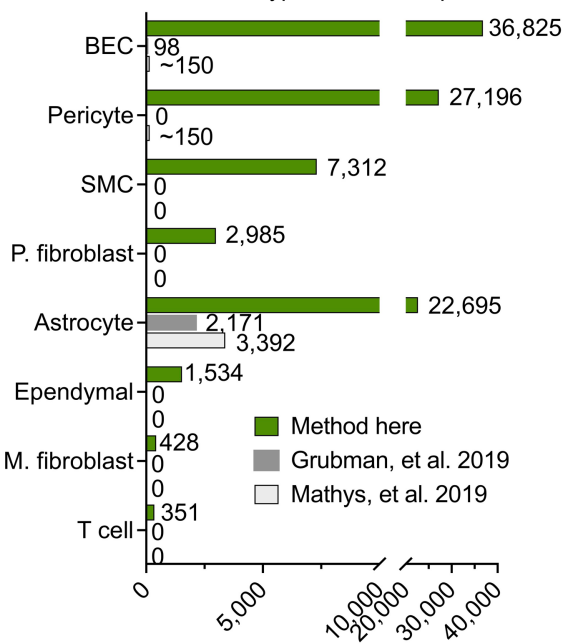

\# of nuclei captured

g Cell type proportions across individuals

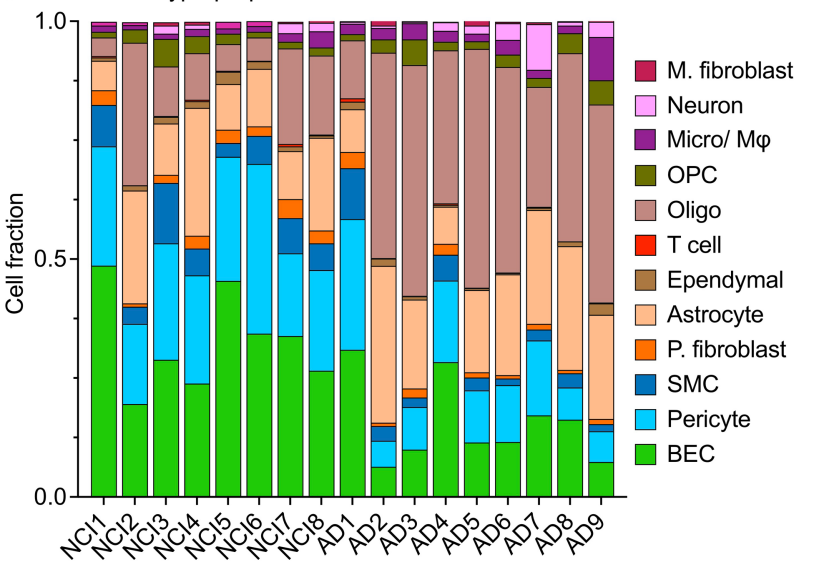

Extended Data Fig. 1 | Enhanced capture and characterization of human brain vascular nuclei. a, Detailed schematic of the VINE-seq method to capture human brain vascular and immune cell types for single-nucleus sequencing. b, Total number of nuclei, median number of unique molecular identifiers (UMI), and median number of genes for each human sample sequenced from hippocampus and superior frontal cortex.c, Quantification of the median number of genes detected per nuclei across groups $(n=17$ hippocampus and $n=8$ cortex; $n=8 \mathrm{NCI}$ and $n=9 \mathrm{AD}$, two-sided $t$-test;
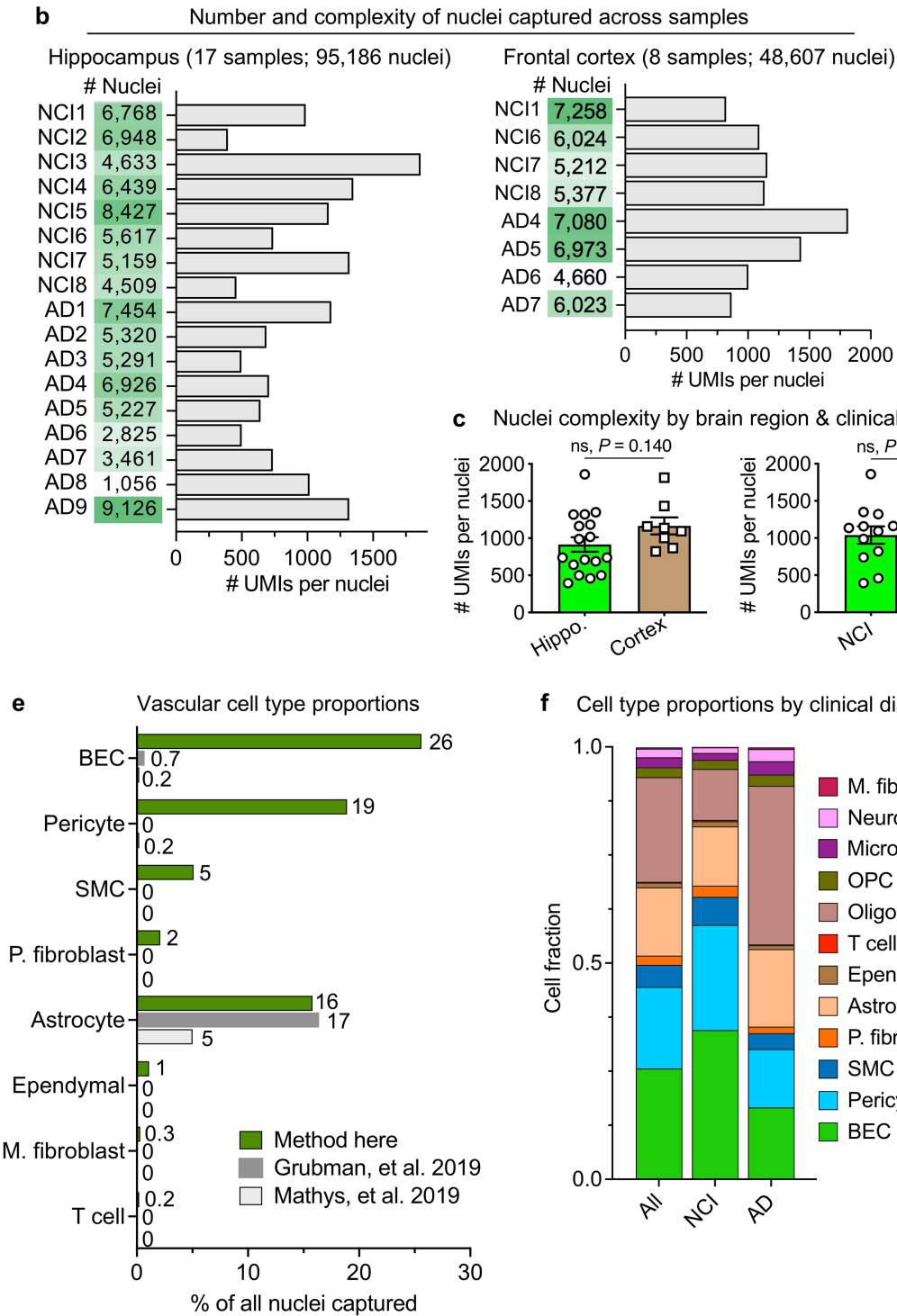

c Nuclei complexity by brain region \& clinical diagnosis
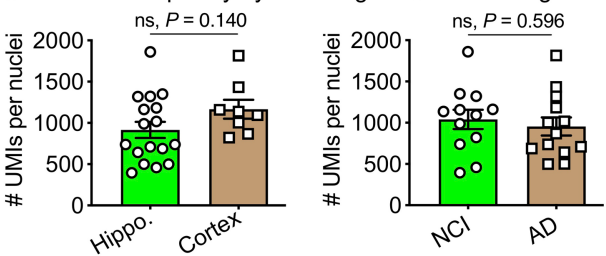

f Cell type proportions by clinical diagnosis

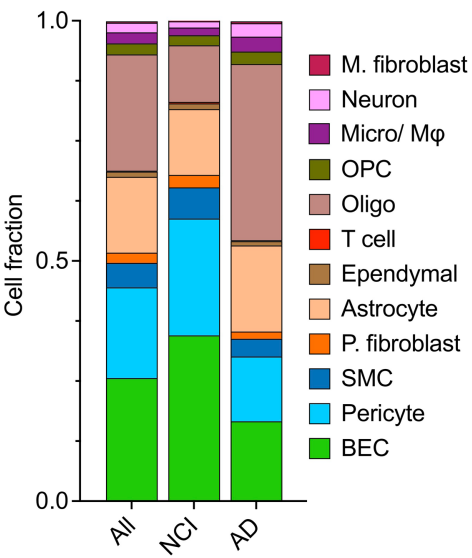

h Cell type proportions and quantification by brain region
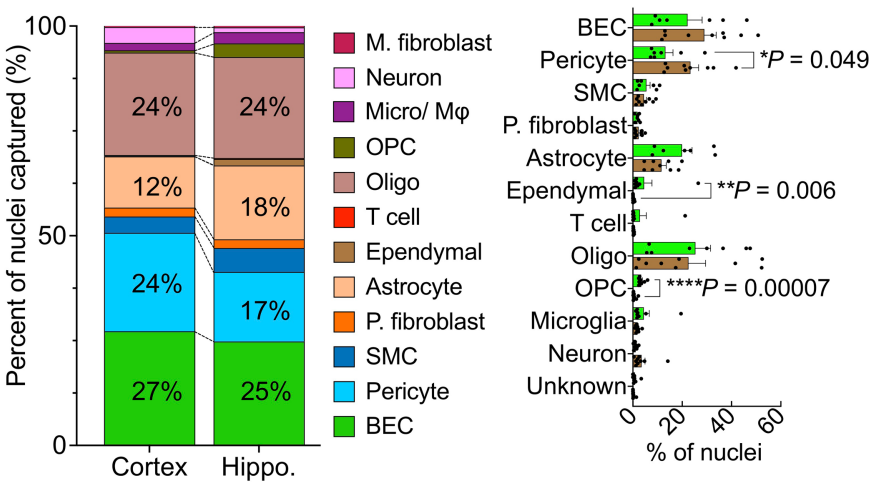

mean+/-s.e.m.).d,e, Quantification of the number (d) and proportion (e) of cerebrovascular cell types captured via the VINE-seq method introduced here compared to recent snRNA-seq studies ${ }^{16,17}$.f, Summary quantification of the proportion of captured cell types, by individuals with $\mathrm{NCI}$ and individuals with AD.g, Quantification of the proportion of captured cell types across individuals.h, Summary (left) and quantification (right) of the proportion of captured cell types by brain region ( $n=17$ hippocampus and $n=8$ cortex; $n=8$ $\mathrm{NCI}$ and $n=9 \mathrm{AD}$, two-sided $t$-test; mean +/-s.e.m.). 
a H\&E staining across $8 \mathrm{NCl}$ patients revealing no significant vascular pathologies

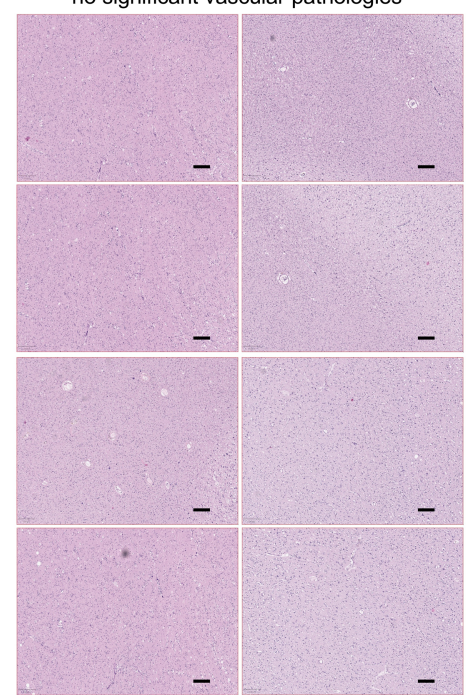

C Cell type marker expression, minimal doublet contamination

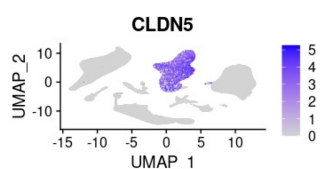

ACTA2

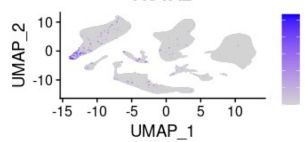

MBP
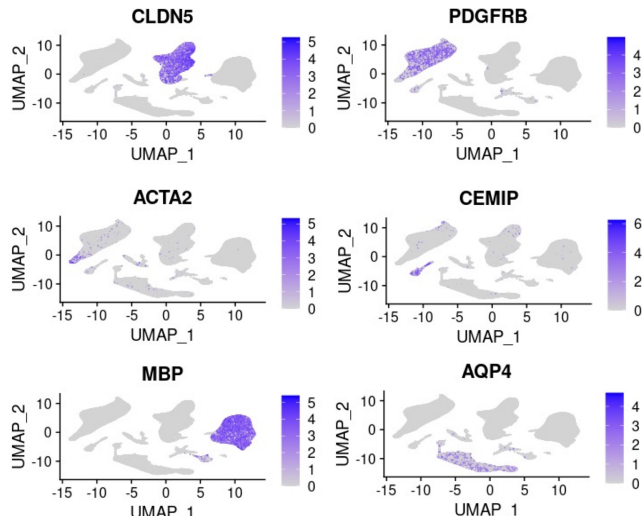

CEMIP

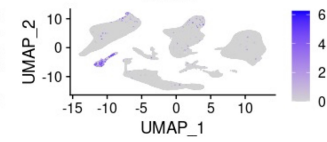

AQP4
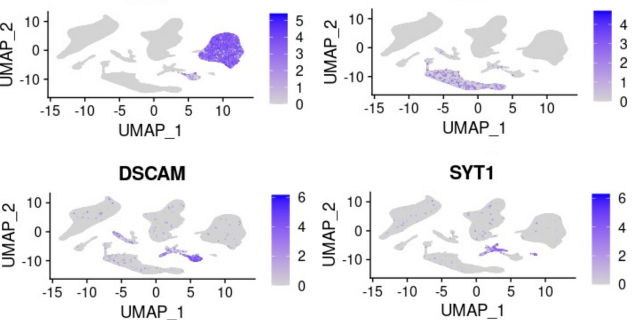

g Human brain T cells are CD8 cytotoxic and CD4 NV/CM
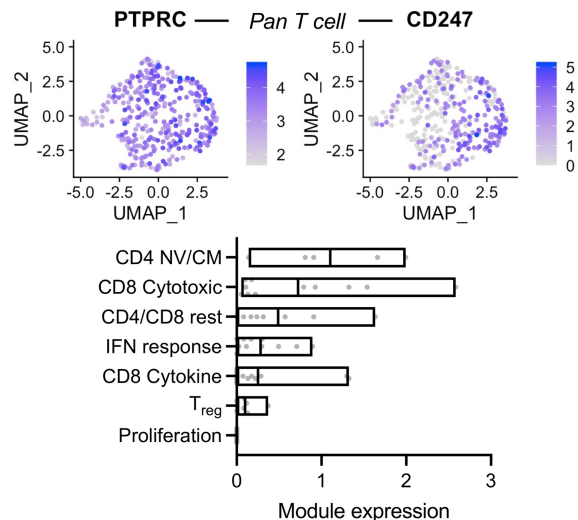

Module expression

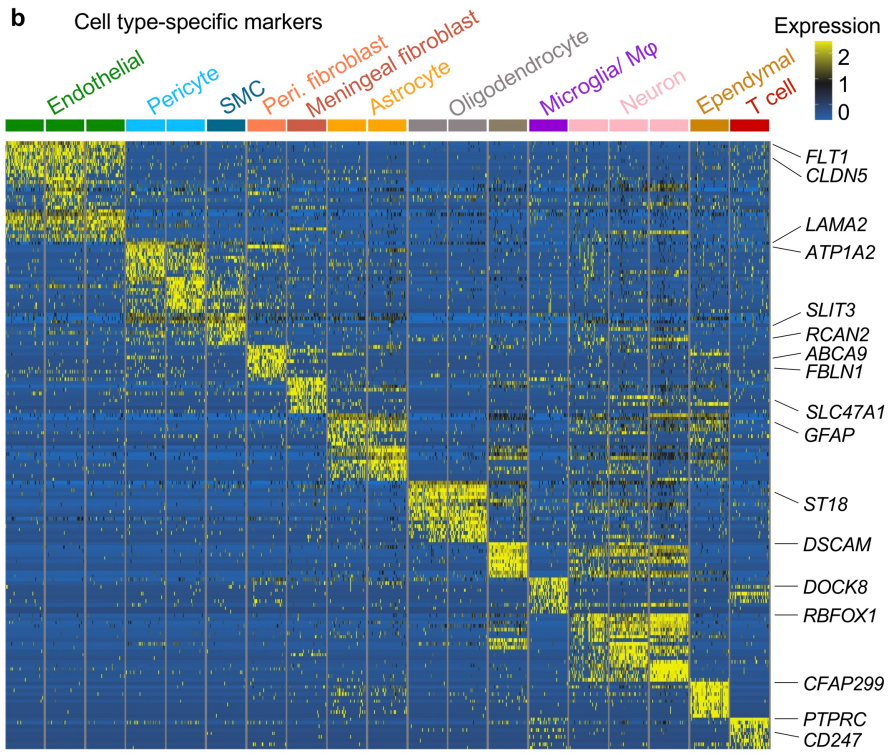

d Microglia and perivascular macrophages subcluster

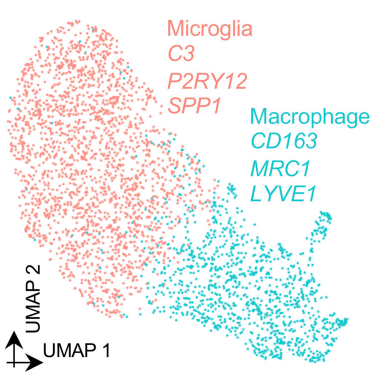

e Microglia and macrophage markers

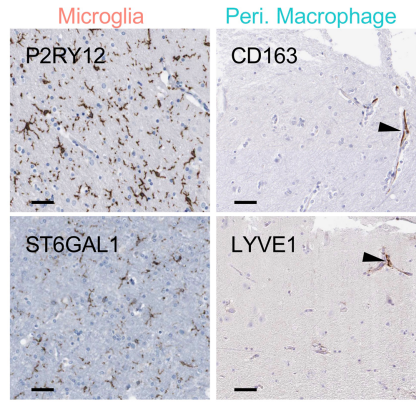

f Differentially expressed human brain myeloid genes \& pathways

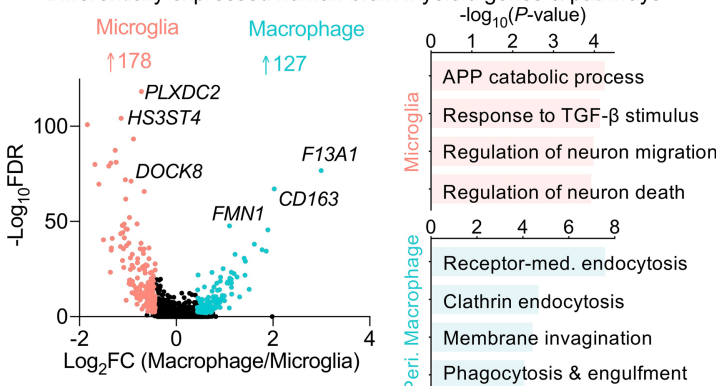

h Human astrocyte heterogeneity is determined

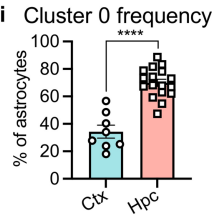

j Cluster 1 frequency

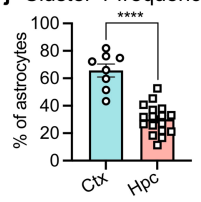

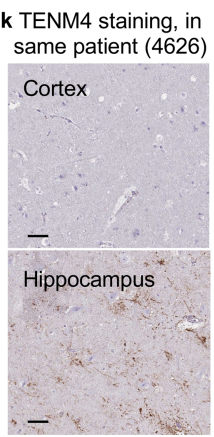

Extended Data Fig. 2 |See next page for caption. 


\section{Article}

Extended Data Fig. 2 | Diversity and heterogeneity of human brain vascular cell types. a, Representative $H \& E$ images used by two neuropathologists to evaluate $\mathrm{NCI}$ cortical and hippocampal tissue for vascular pathology. No significant vascular pathology was observed. Scale bars, $200 \mu \mathrm{m}$. b. Discovery of the top cell-type-specific marker genes across the major classes of cells captured. The colour bar indicates gene expression from low (blue) to high (yellow).c, Validation of cell-type annotations and confirmation of minimal doublet contamination using established cell-type markers. d, UMAP projection of captured myeloid cells, forming two distinct clusters corresponding to parenchymal microglia and brain barrier macrophages. Example marker genes listed. e, Immunohistochemical validation of microglial and perivascular macrophage markers. Scale bars, $50 \mu \mathrm{m}$. Image credit: Human Protein Atlas ${ }^{25,74}$ (http://www.proteinatlas.org).f, Global view of DEGs comparing human brain macrophages and microglia (left, MAST, Benjamini-
Hochberg correction; FDR $<0.01$ and $\log \mathrm{FC}>0.5\left[\log _{2} \mathrm{FC}>0.72\right]$ to be coloured significant). Pathways enriched in microglia versus macrophages (right), recapitulating interesting biology such as the unique TGF- $\beta$ molecular signature in microglia ${ }^{75}$.g, Expression of top gene markers for various T cell subtypes (top), and quantification of their expression as a module (bottom) ${ }^{76}$. Brain T cells exhibit highest expression of markers corresponding to CD8 cytotoxic and CD4 Naive/Central memory (NV/CM) T cells. h, UMAP projection of captured astrocytes, forming two distinct clusters, and split by brain region. Example marker genes listed. $\mathbf{i}-\mathbf{j}$, Quantification of astrocyte cluster $\mathbf{0}(\mathbf{b})$ and 1 (c) frequency in the cortex and hippocampus ( $n=8$ cortex and $n=17$ hippocampus, Mann-Whitney $t$-test; mean $+/$-s.e.m.). k, Immunohistochemical validation of the brain region-specific astrocyte marker TENM4. Scale bars, $50 \mu \mathrm{m}$. 

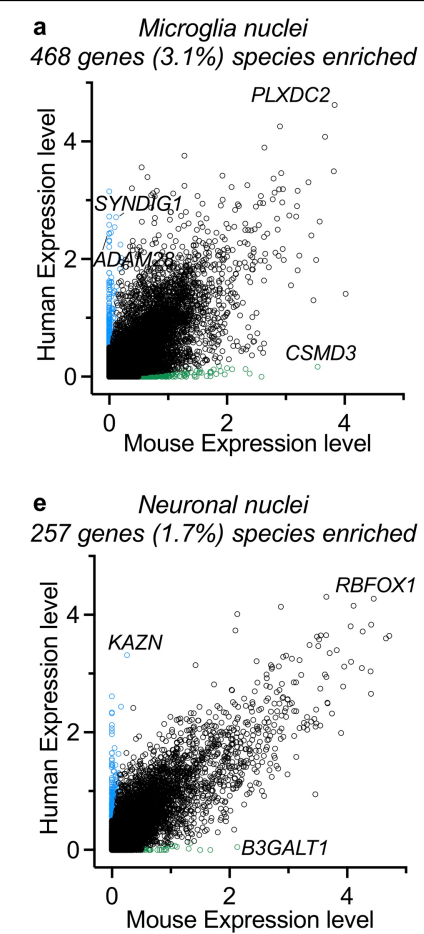

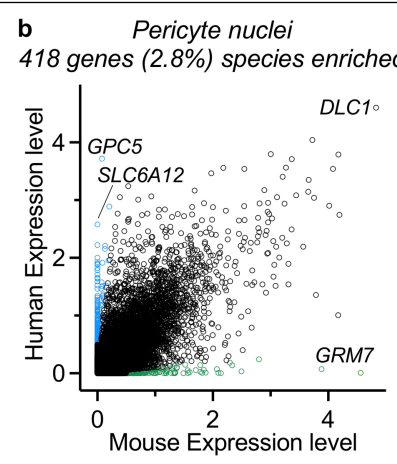

f

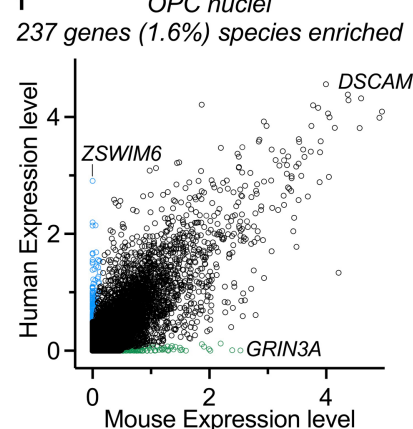

c Oligodendrocyte nuclei

332 genes (2.2\%) species enriched

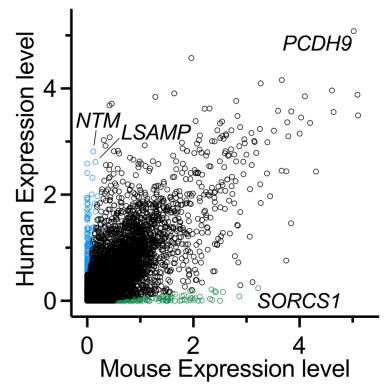

Astrocyte nuclei

316 genes $(2.1 \%)$ species enriched

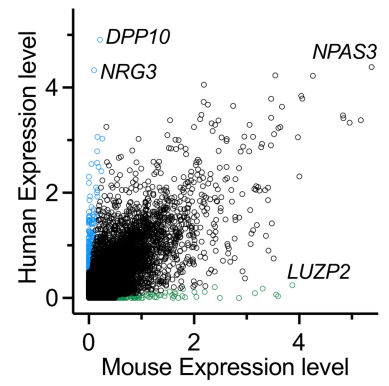

g

g Examples of human-enriched vascular-expressed genes
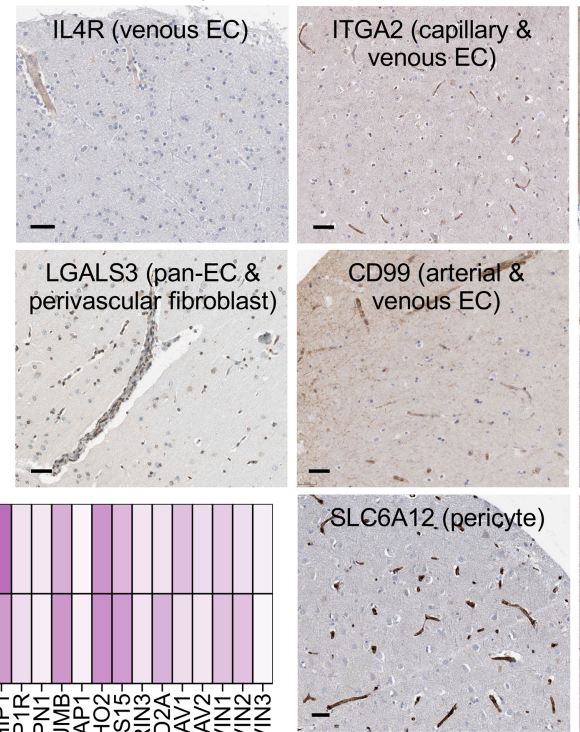

CASP10 (pan-EC)

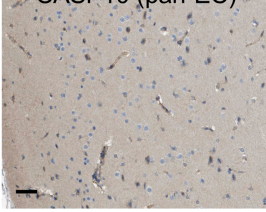

CD55 (arterial EC)

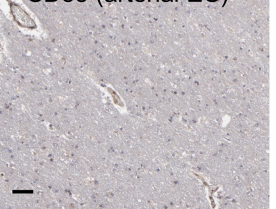

EFHD1 (mural)

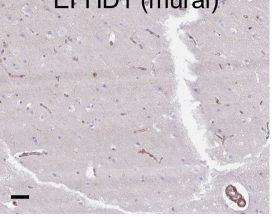

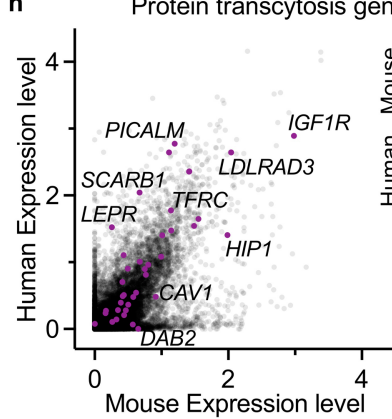
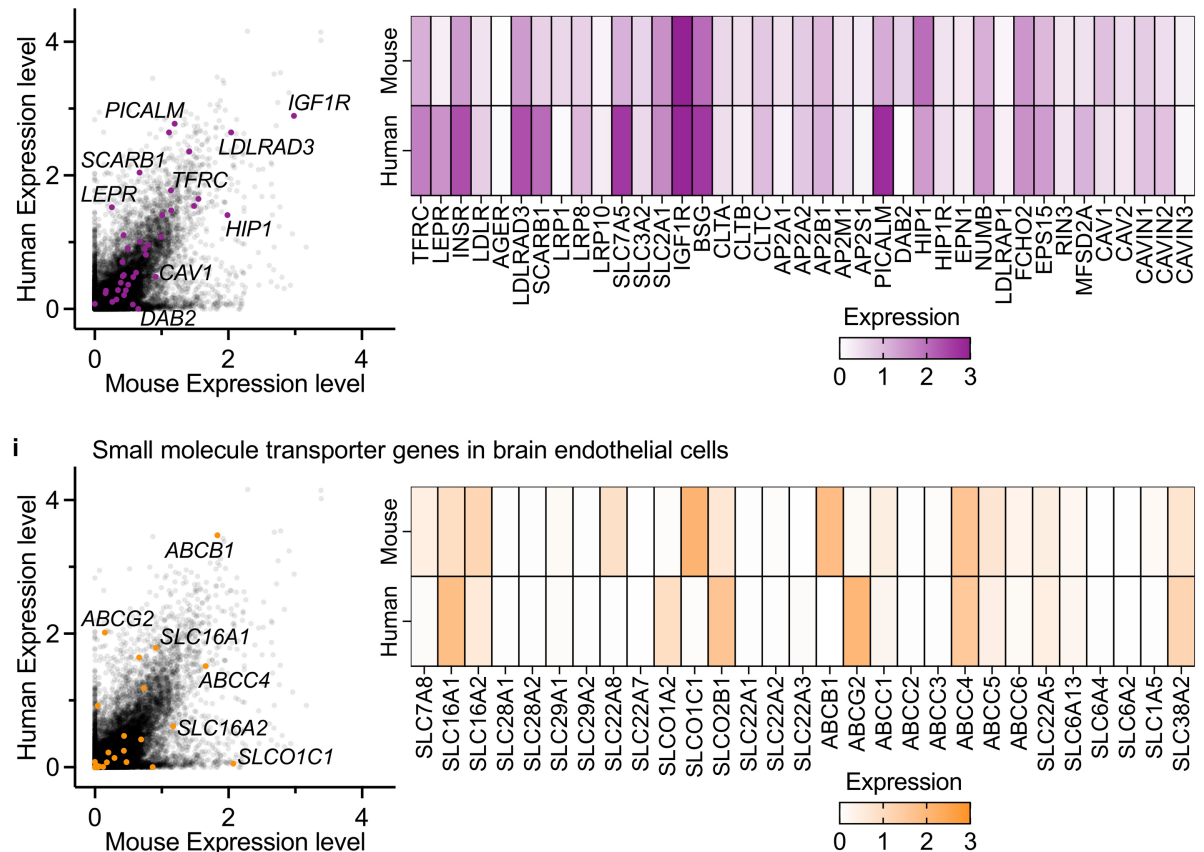

Extended Data Fig. 3 | Species-specific gene expression across brain cell types and their pharmacological relevance. $\mathbf{a}-\mathbf{f}$, Identification of species-specific genes. Both mouse and human transcriptomes were generated and analysed similarly via single-nucleus RNA-sequencing. Mice were 19 months of age to match the average age of our human cohort. Species-specific/enriched are coloured.g, Immunohistochemical confirmation of genes predicted to be enriched or specific to human cerebrovascular cells compared to mouse (isolated mouse nuclei and per Vanlandewijck, et al., 2018) ${ }^{12}$, in terms of overall expression or zonation. In parenthesis is the cell type predicted to be uniquely or exhibiting enriched expressed in human over mouse. Scale bars, $50 \mu \mathrm{m}$. Image credit: Human Protein Atlas ${ }^{25,74}$ (http://www.proteinatlas.org). h-i, Mouse and human BEC expression of genes mediating protein transcytosis $(\mathbf{h})$ and small molecule influx and efflux (i). 
a Human brain vascular expression of genes relevant to SARS-CoV-2 brain entry

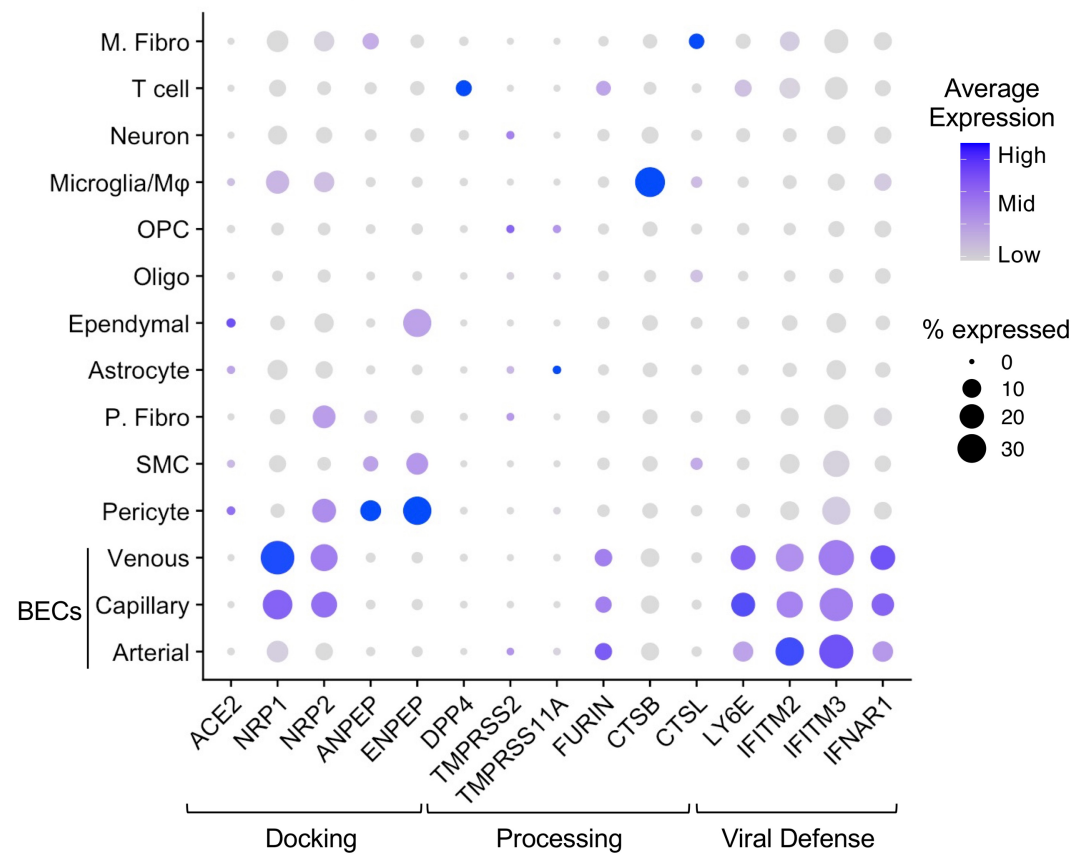

b SPP1 expression in human microglia \& oligodendrocytes

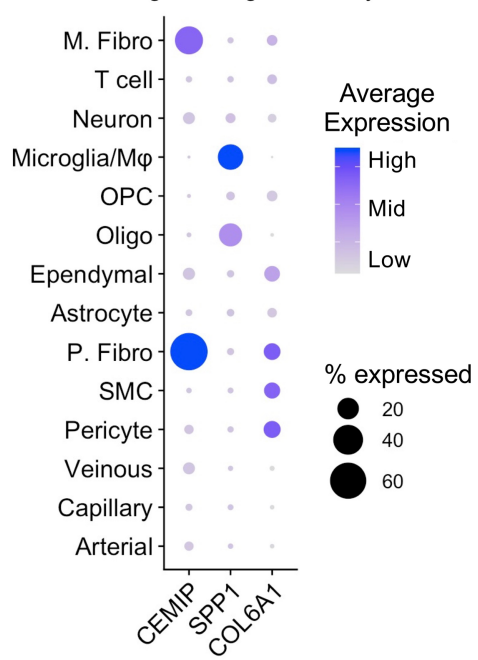

C No expression of immuno-oncology target CD19 across 34,508 human cortical and hippocampal pericytes and SMCs

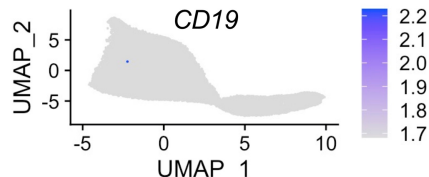

Extended Data Fig. 4 | Human brain vascular expression of genes relevant to disease. a, Brain vascular expression of genes relevant to SARS-CoV-2 brain entry, as summarized in ladecola, et al. $2020^{77}$. b. Expression of the mouse perivascular fibroblast-like gene $S p p 1$ is instead specifically expressed in human myeloid cells and oligodendrocytes $(S P P 1, \text { top })^{78} \cdot \mathbf{c}$, No expression of the
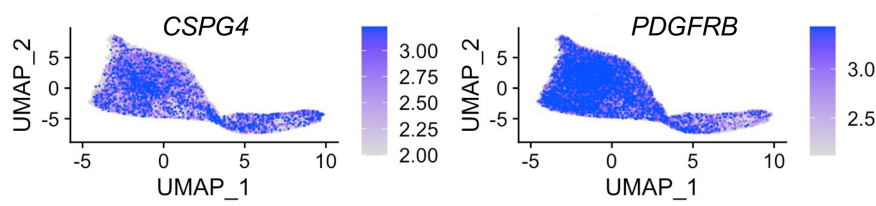

immuno-oncology target $C D 19$ and its chaperone $C D 81$ across human adult brain pericytes and $\mathrm{SMCs}^{79}$. Note: cells with any finite expression are ordered to the front to ensure all expression is visible, but this carries the potential to visually overestimate average expression. 

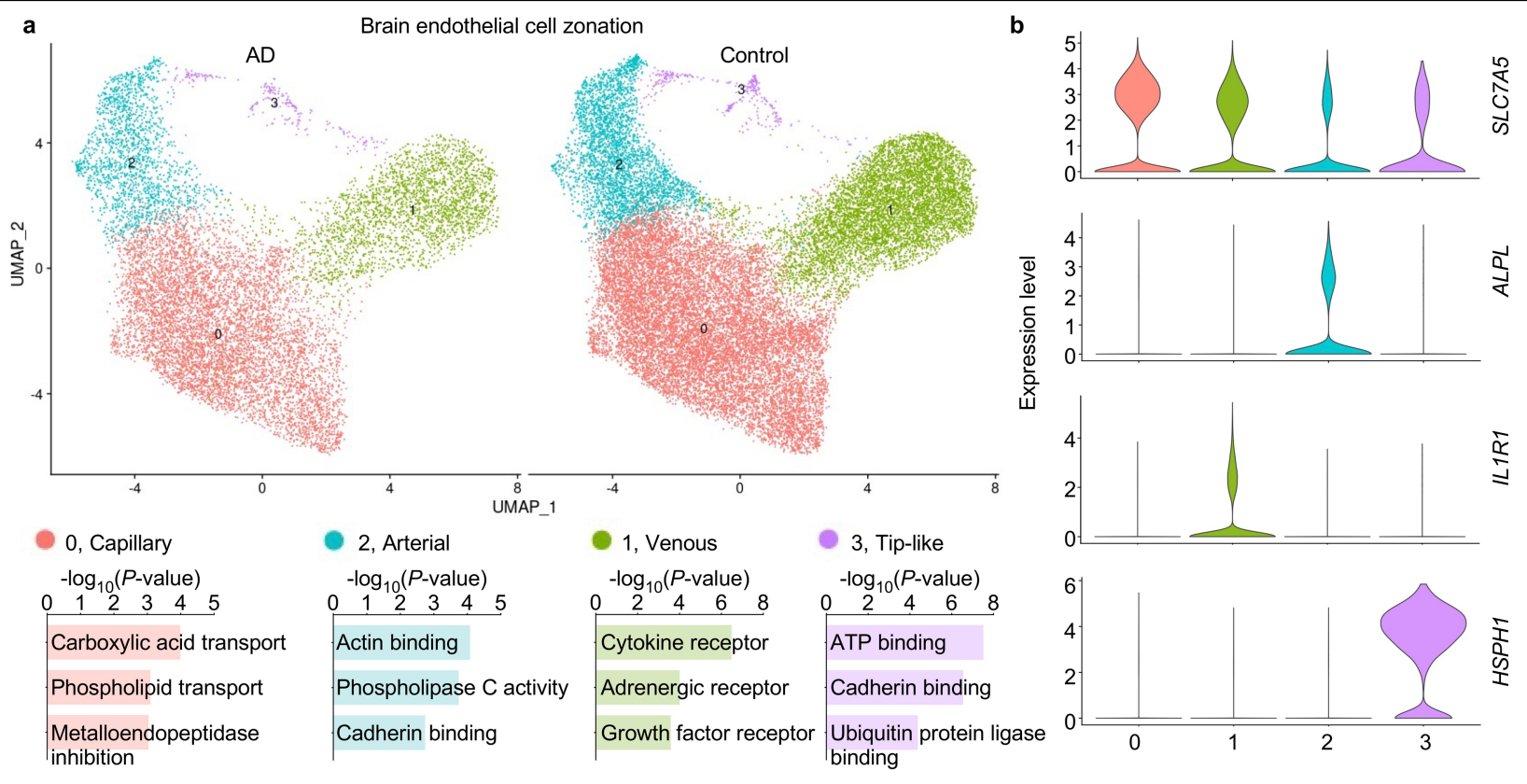

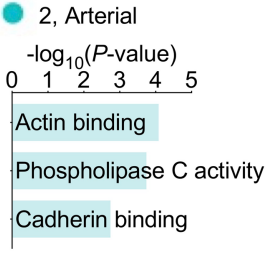
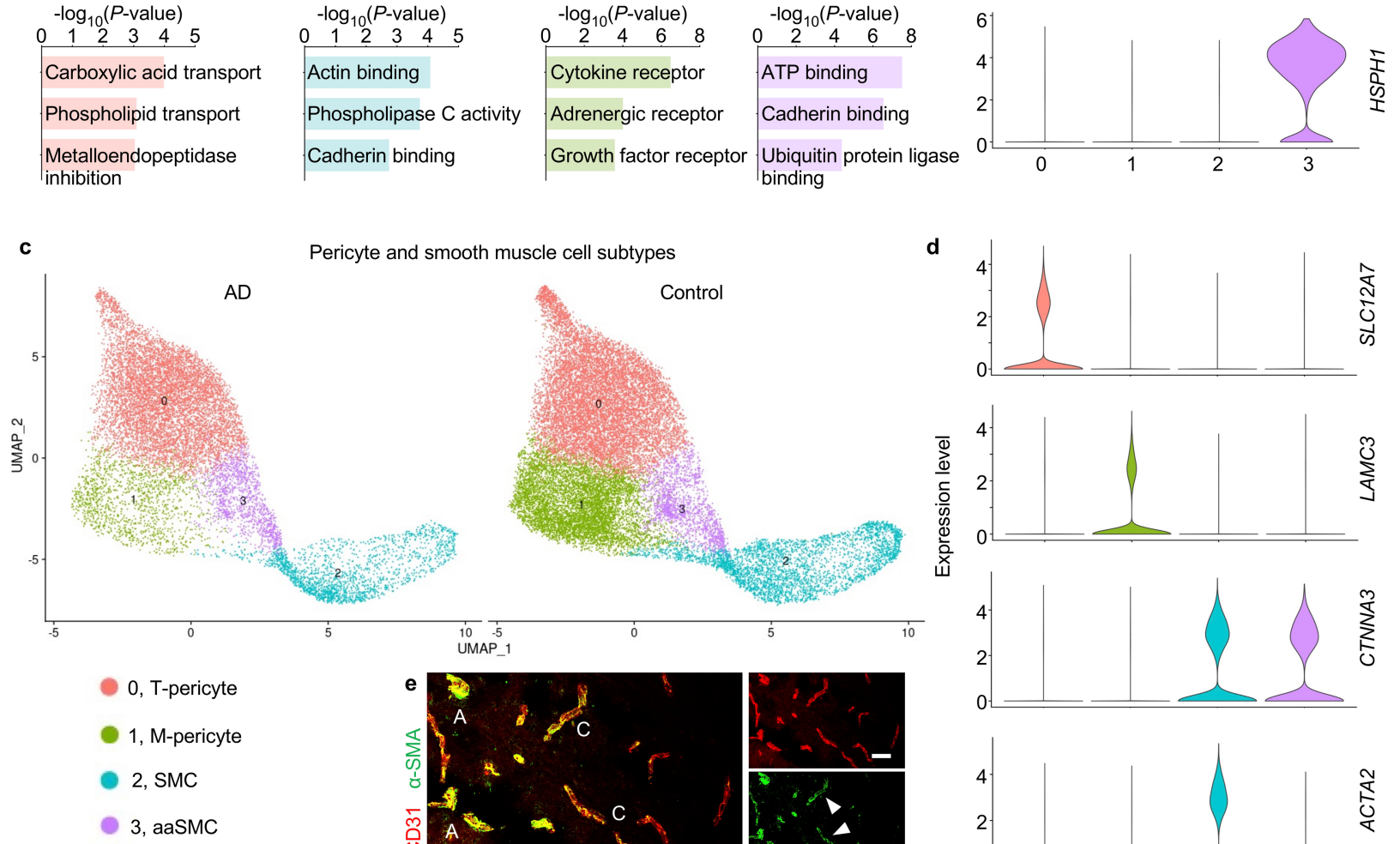

Pericyte and smooth muscle cell subtypes

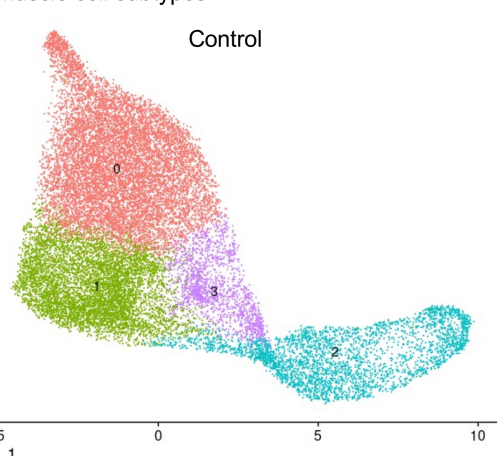

d
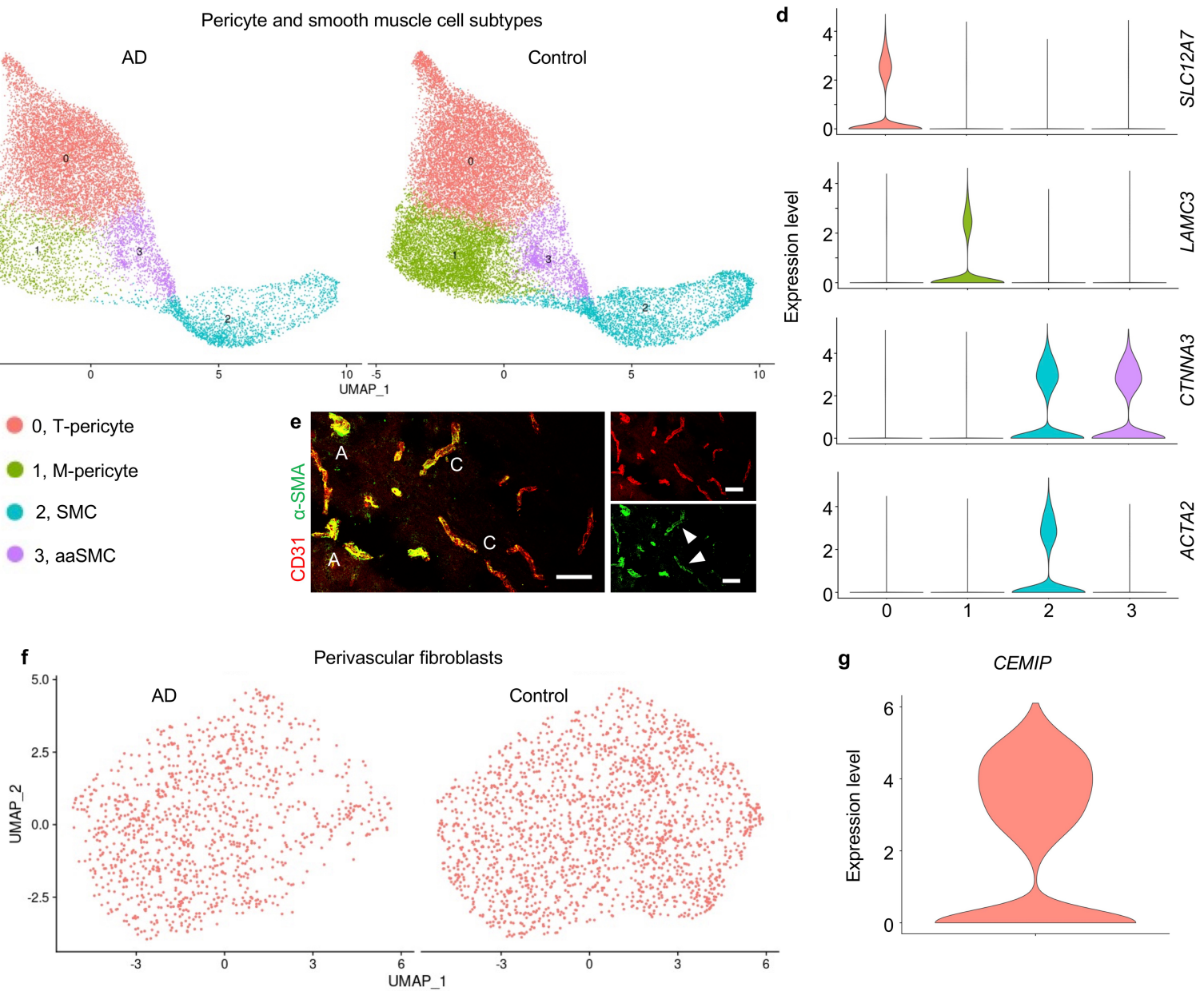

g

CEMIP

Extended Data Fig. 5 | Brain endothelial and mural cell zonation and subpopulations. a, UMAP projection of captured BECs, organizing by arteriovenous zonation. Bottom, tip cell markers expressed in the tip-like/ proteostatic EC cluster. $\mathbf{b}$, Validation of BEC zonation clusters using established zonation markers ${ }^{12}$. Violin plots are centred around the median, with their shape representing cell distribution. $\mathbf{c}, \mathbf{d}$, As in $\mathbf{a}, \mathbf{b}$ but for pericytes
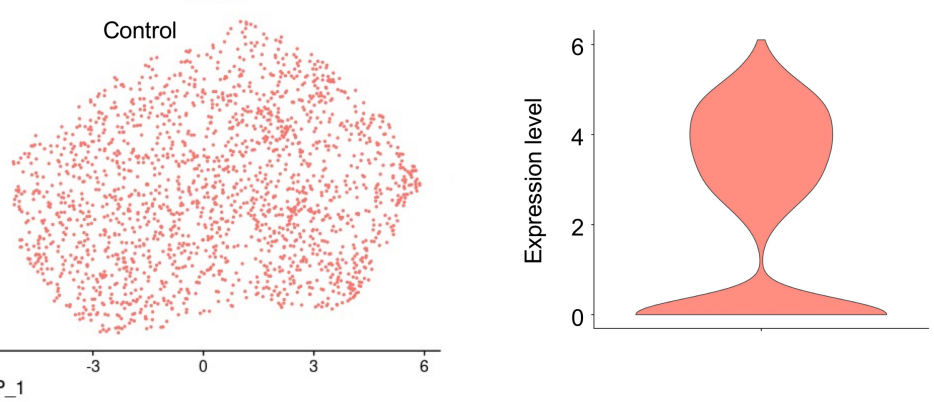

and SMCs. Note that the anatomical locations of pericyte 0 and 1 have not yet been determined. e, Immunohistochemical validation of ACTA2 ( $\alpha$-SMA) expression in human SMCs and less so in capillary pericytes. A denotes arterial and $\mathrm{C}$ denotes capillary. Arrowheads specify capillary pericytes expressing ACTA2. Scale bars, $50 \mu \mathrm{m} . \mathbf{f}, \mathbf{g}$, As in $\mathbf{a}, \mathbf{b}$ but for perivascular fibroblast-like cells, as recently discovered in mice ${ }^{12}$. 


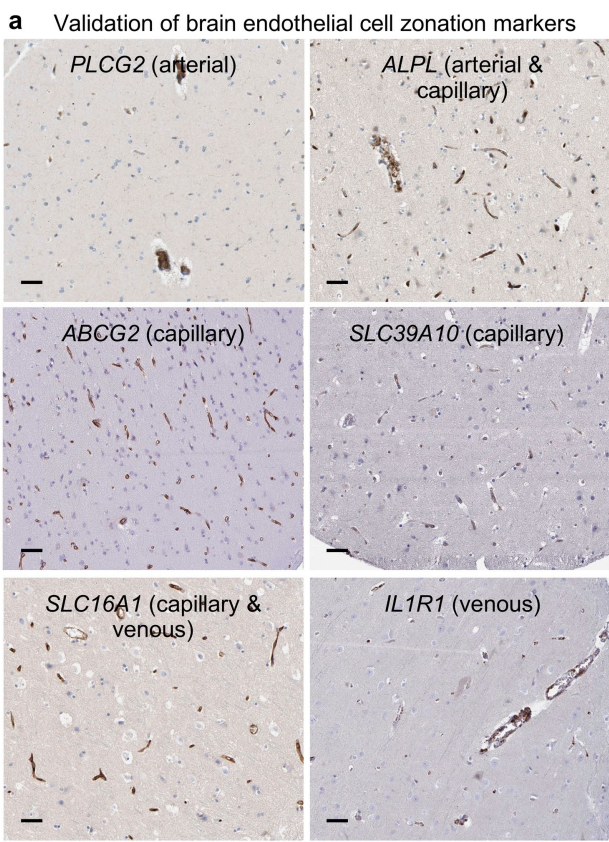

c Human capillary expression of mouse venous markers

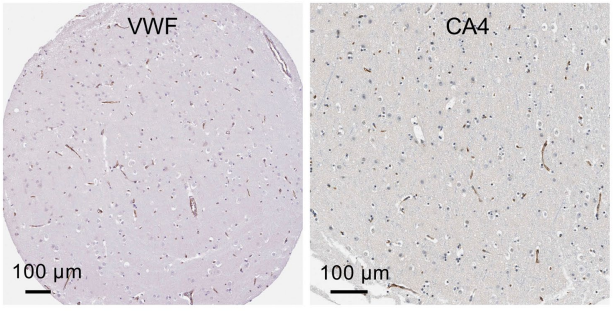

d Validation of brain mural cell markers

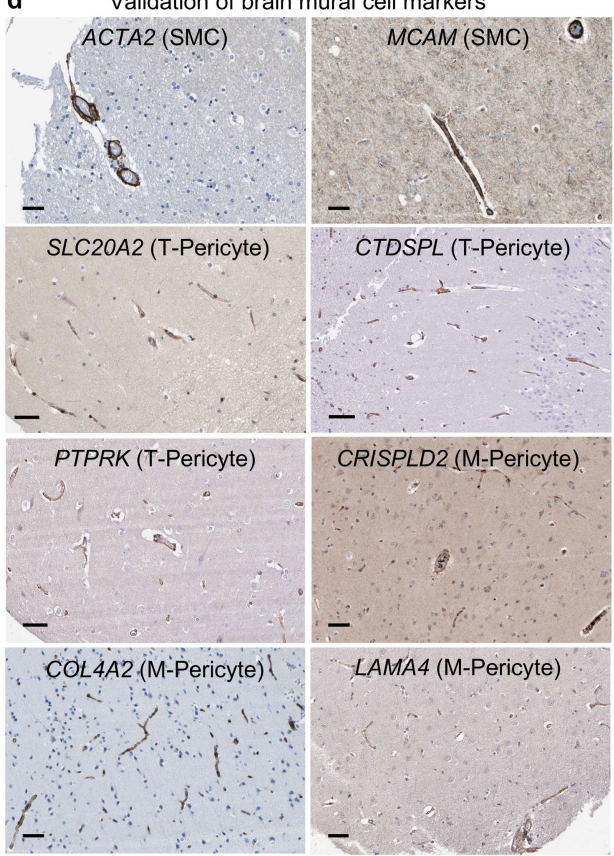

Extended Data Fig. 6 | Brain endothelial zonation and mural cell subtype markers. a, Immunohistochemical validation of zonation and cell subtype markers in BECs. Scale bars, $50 \mu \mathrm{m}$. Image credit: Human Protein Atlas ${ }^{25,74}$ (http://www.proteinatlas.org).b,Comparison of the zonal specificity of genes in arterial, capillary, and venous cells. Axis plot a specificity score, as defined in the Methods. For example, specificity score for capillaries $=\operatorname{avg}(\operatorname{logFC}$ (cap/ven), logFC(cap/art)).c, Immunohistochemical validation of capillary
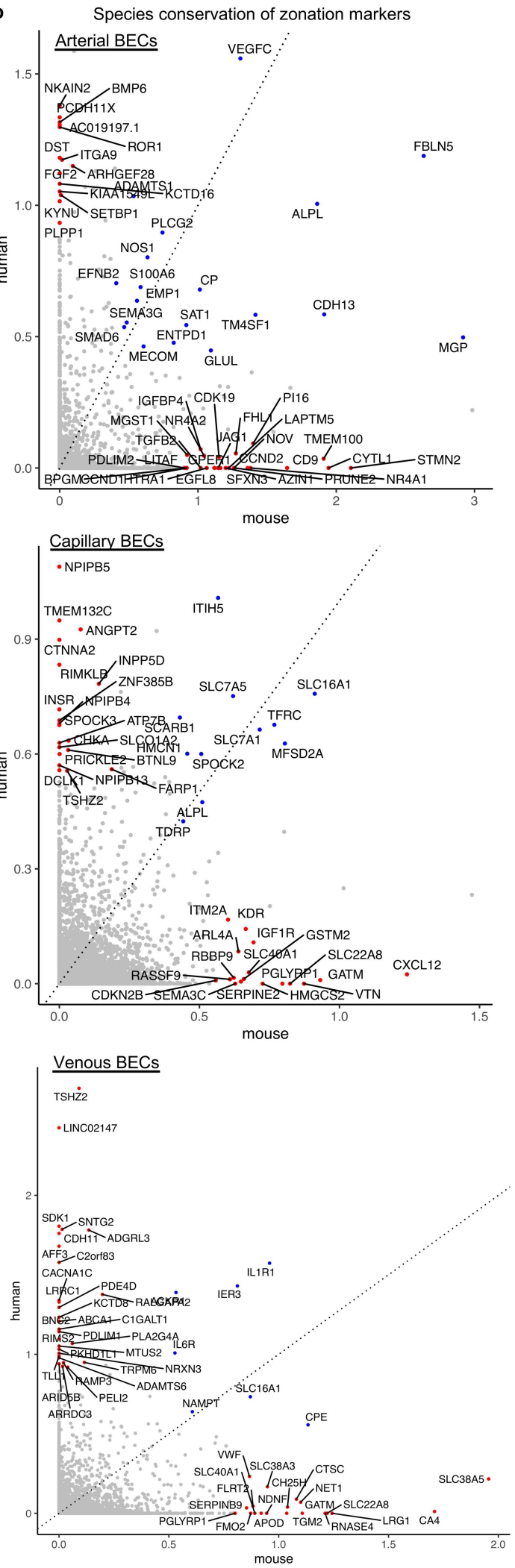

expression in human brains of the mouse venous-specific marker VWF and CA4, with similar patterns observed across multiple primary antibody clones. Scale bars, $100 \mu \mathrm{m}$. Image credit: Human Protein Atlas ${ }^{25,74}$ (http://www. proteinatlas.org). d, Immunohistochemical validation of zonation and cell subtype markers in brain SMCs and pericytes. Scale bars, $50 \mu \mathrm{m}$. Image credit: Human Protein Atlas ${ }^{25,74}$ (http://www.proteinatlas.org). 


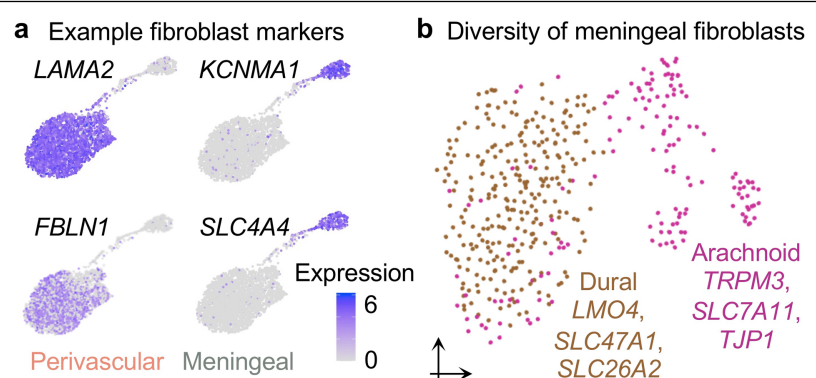

d

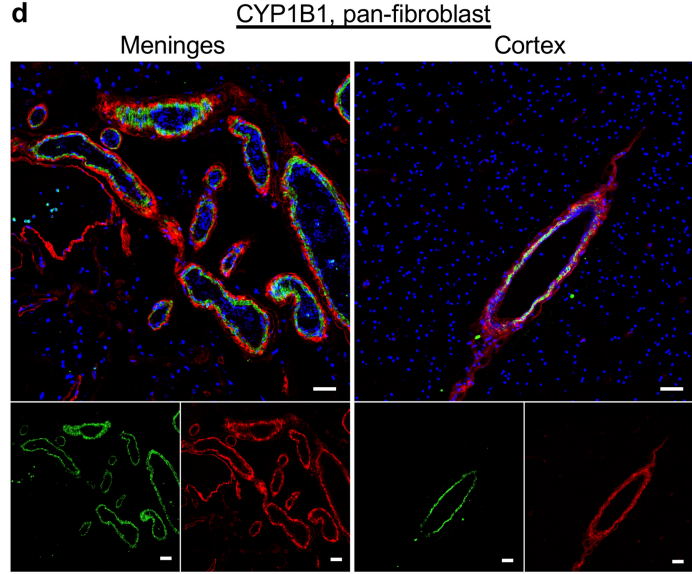

CYP1B1 Collagen IV Hoechst

\section{Extended Data Fig. 7 |Specialization and functions of human brain}

fibroblasts. a, Expression of example markers demarcating perivascular from meningeal fibroblasts. b, UMAP of 428 meningeal fibroblast nuclei, subclustering into anatomically segregated dural and arachnoid space fibroblasts. c, Expression of the genes constituting the major fibrotic scar component collagen I in pericytes and fibroblasts. Collagen I is composed of two components, COL1A1 and COL1A2. Column annotations: T-PC = solute transport pericyte and $\mathrm{M}-\mathrm{PC}=$ Extracellular matrix regulating pericyte, P. $\mathrm{FB}=$ Perivascular fibroblast, and M. FB = Meningeal fibroblast. d, e, Protein
C Expression of fibrotic scar genes encoding Collagen I in perivascular fibroblasts

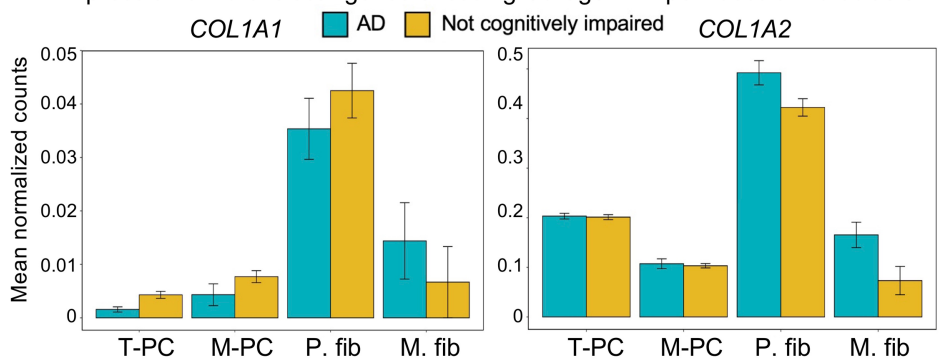

e SLC47A1, Meningeal fibroblast-specific

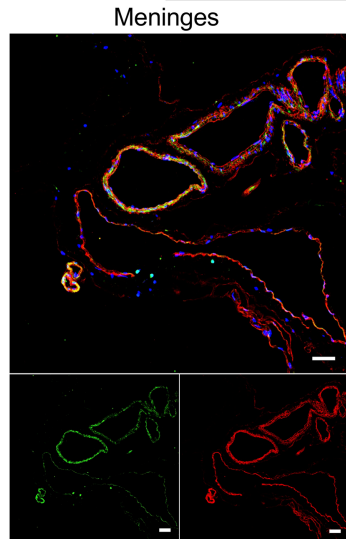

SLC47A1 Collagen IV Hoechst f Perivascular fibroblast, human vs mouse markers
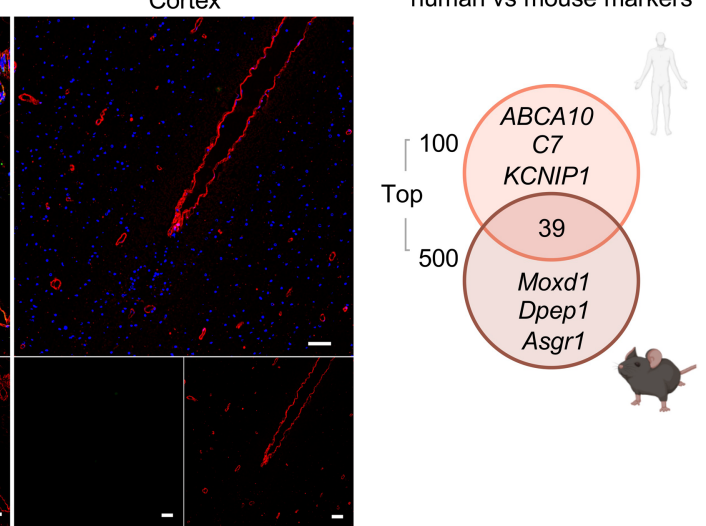

immunostaining validation of polarized expression of human brain meningeal and perivascular fibroblast pumps: the common marker CYP1B1 (d, serves as a control) and the meningeal fibroblast-specific influx pump SLC47A1 (e). Scale bars, $50 \mu \mathrm{m}$. f, Overlap between the top 100 perivascular fibroblast-like cell markers and those identified in mice. A more lenient set of 500 (instead of 100) mouse markers ${ }^{80}$ were used for comparison to ensure claims of species-specificity were robust. Note: the species-conservation of a cell-type marker depends on species-specific changes in the given cell type and changes amongst the remaining background cell types. 
a Confirmation of presence \& absence of $A D$ pathology by subject group

Hoechst $A B$ Thioflavin-S
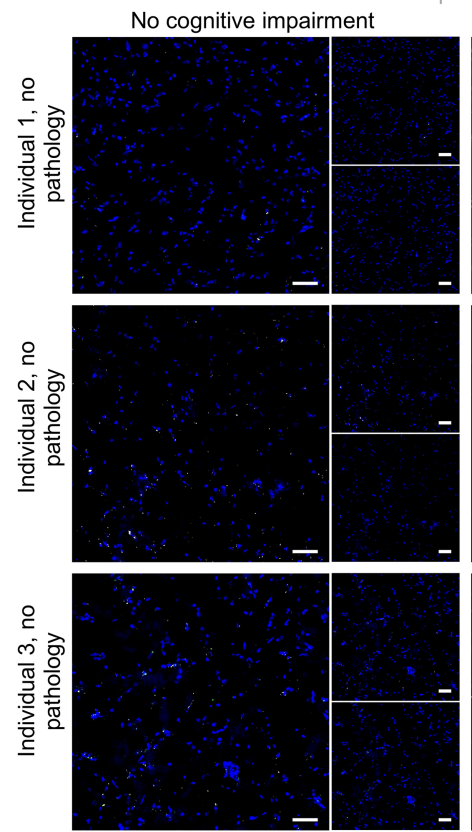

e Vascular density quantification controls
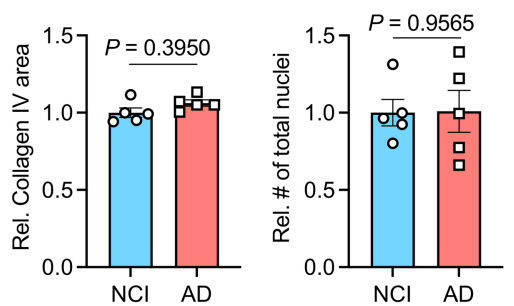

h Number of AD DEGs by pericyte subtype

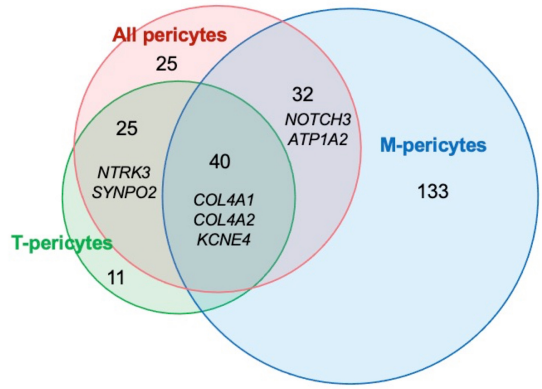

I Regional vascular cell abundances in $A D$ $\square$ Control Hpc $\square$ AD Hpc $\square$ Control Ctx $\square$ AD Ctx
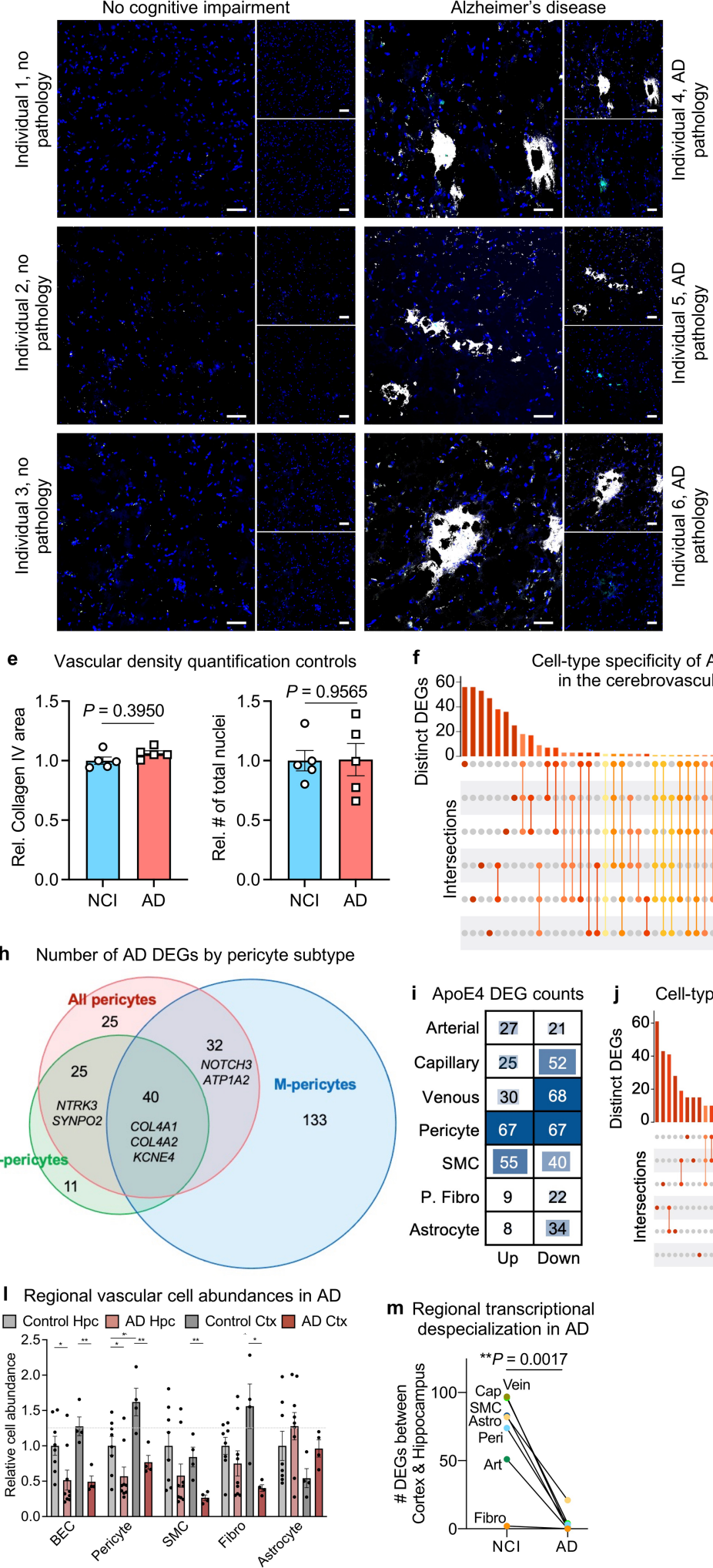

f Cell-type specificity of AD DEG
in the cerebrovasculature

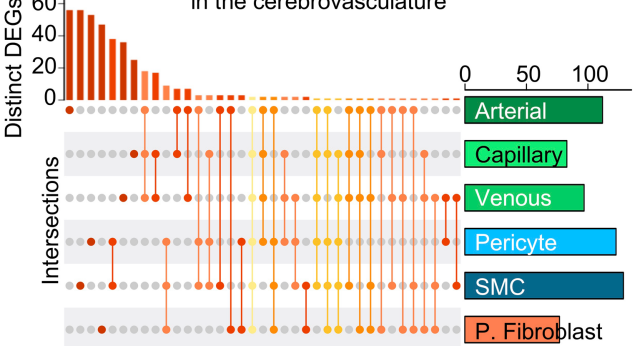

g
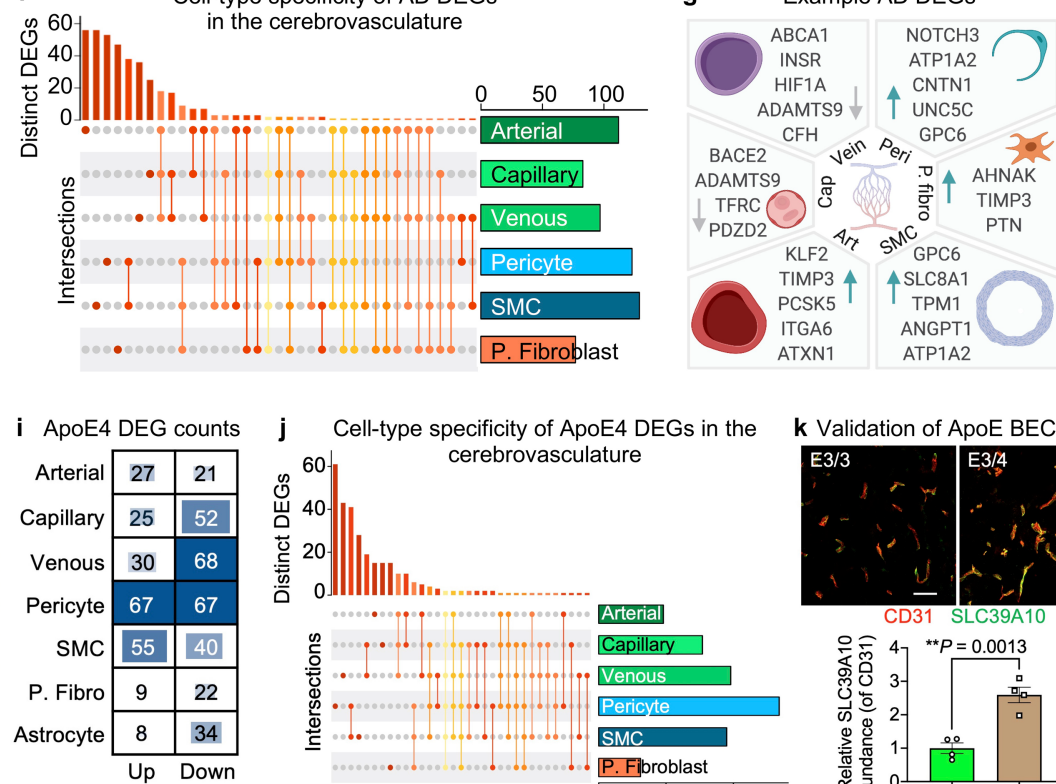

b Total Amyloid $\beta$

C Cored \& neuritic
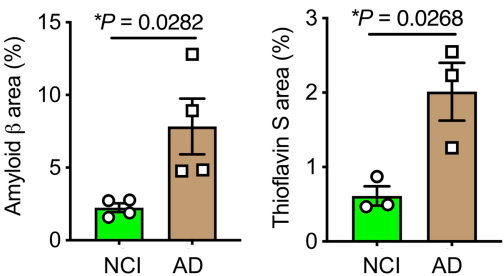

d Human vascular cell representation in $A D$

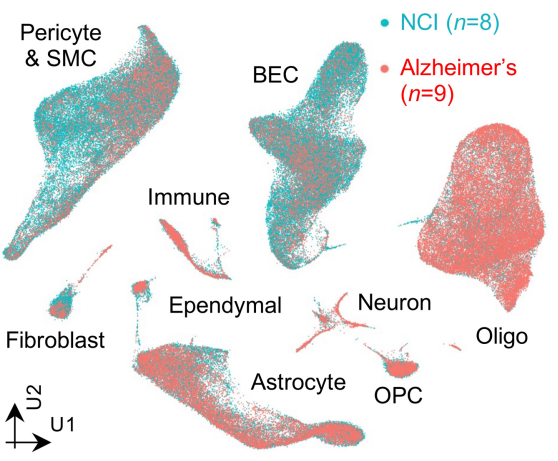

$\mathbf{k}$ Validation of ApoE BEC DEG
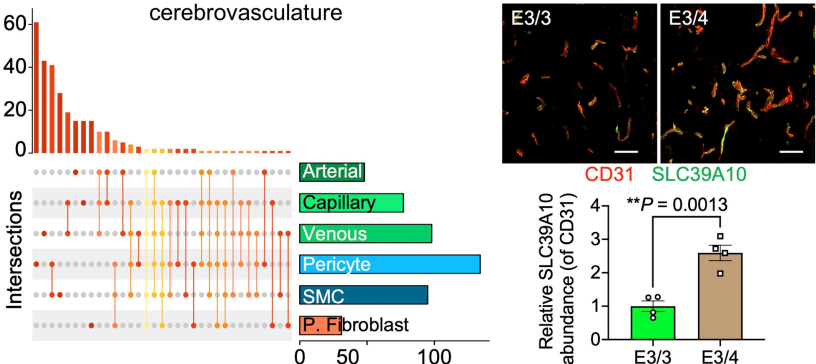

운도 $47{ }^{* *} P=0.0013$

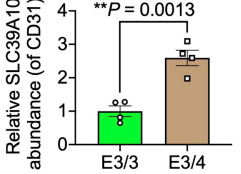

m Regional transcriptional despecialization in $\mathrm{AD}$

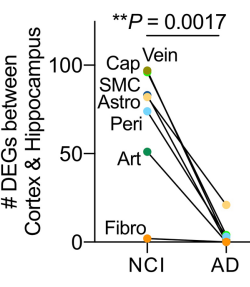

Extended Data Fig. 8 |See next page for caption. 
Extended Data Fig. 8 | Vascular cell-type-specific perturbations in patients with AD and ApoE4 carriers. a, Immunohistochemistry with anti- $\beta$-amyloid antibody (D54D2, white), Thioflavin S (green), and Hoechst (blue) in the hippocampus of individuals with $\mathrm{NCl}$ and individuals with AD. Scale bars, $40 \mu \mathrm{m}$. b, Quantification of $\beta$-amyloid immunostaining in a for overall $\beta$-amyloid $(n=4$ $\mathrm{NCl}$ and $\mathrm{AD}$, two-sided $t$-test; mean $+/$-s.e.m.).c, As in $\mathbf{b}$ but for cored and neuritic $\beta$-amyloid plaques ( $n=3 \mathrm{NCl}$ and $\mathrm{AD}$, two-sided $t$-test; mean $+/$-s.e.m.).d, UMAP of 143,793 nuclei captured from 17 human hippocampus and superior frontal cortex samples, coloured by AD diagnosis. e, Quantification controls for Fig. 5 b. Quantification of Collagen IV ${ }^{+}$vasculature (left) and number of total (regardless of Collagen $\mathrm{IV}^{+}$overlap) Hoechst ${ }^{+}$nuclei $(n=5 \mathrm{NCI}$ and $\mathrm{AD}$, nested two-sided $t$-test; mean $+/$-s.e.m.).f, Matrix layout for intersections of AD DEGs shared across and specific to each cell type. Circles in the matrix indicate sets that are part of the intersection, showing that most DEGs are cell-type-specific. g, Example DEGs in AD: arterial (Art), capillary (Cap), venous (Vein), pericyte (Peri), perivascular fibro blast-like cell (P. fibro), and SMC. Blue arrow indicates upregulated and grey arrow downregulated genes. $\mathbf{h}$, Summary of the number of AD DEGs by pericyte class: $T-, M-$, and all pericytes combined to evaluate DEGs that could arise due to a disproportionate loss of M-pericytes in AD. i, DEG counts for each cell type in
ApoE4 carriers ( $n=5 \mathrm{ApoE} 3 / 3, n=11 \mathrm{ApoE} 3 / 4$ or ApoE4/4): arterial (Art), capillary (Cap), venous (Vein), pericyte (Peri), perivascular fibro blast-like cell (P. fibro), and SMC. The intensity of the blue colour and the size of the squares are proportional to entry values. $\mathbf{j}$, Matrix layout for intersections of ApoE4 DEGs shared across and specific to each cell type. Circles in the matrix indicate sets that are part of the intersection, showing that most DEGs are cell-type-specific. k, Immunohistochemical validation of the predicted upregulated antiinflammatory DEG SLC39A1O in venous BECs of ApoE4 carriers. Scale bars, $50 \mu \mathrm{m}$ ( $n=4$ ApoE3/3 and ApoE4 carriers, nested two-sided $t$-test; mean +/-s.e.m.).1 ,Among patients with both hippocampus and superior frontal cortex profiled ( $n=4 \mathrm{NCl}$ and $n=4 \mathrm{AD}$ ), quantification of the relative abundance of major vascular cell types ( $\mathrm{NCl}$ hippocampus set as reference, unpaired two-sided $t$-test; mean + - s.e.m.). ${ }^{*} \mathrm{BEC} P=0.0260,{ }^{*} \mathrm{BEC} \mathrm{P}=0.0023,{ }^{*}$ Pericyte $\mathrm{P}(\mathrm{left})=0.0357$, ${ }^{*}$ Pericyte $\mathrm{P}(\mathrm{mid})=0.0237,{ }^{* *}$ Pericyte $\mathrm{P}=0.0077,{ }^{* *} \mathrm{SMCP}=0.0075,{ }^{*}$ Fibroblast $P=0.0109,{ }^{*}$ Astrocyte $P=0.0357 . \mathrm{m}$, As in (I), but comparison of the number of DEGs between brain regions for each cerebrovascular cell type. Analysis done separately for $\mathrm{NCI}$ and AD samples ( $n=7$ cell types, unpaired two-sided $t$-test; mean+/-s.e.m.). 
a Heterogeneous expression of AD GWAS genes within T- and M-pericyte subpopulations

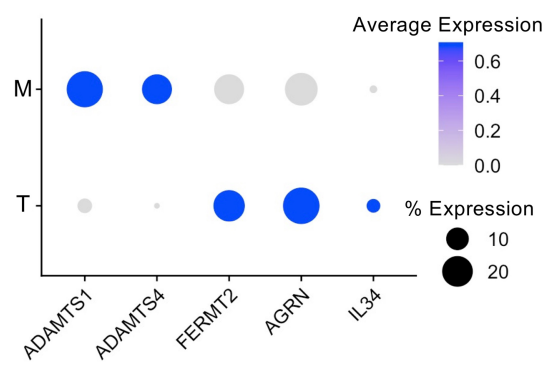

d Difference in proportional expression of top $A D$ GWAS genes between the hippocampus and cortex

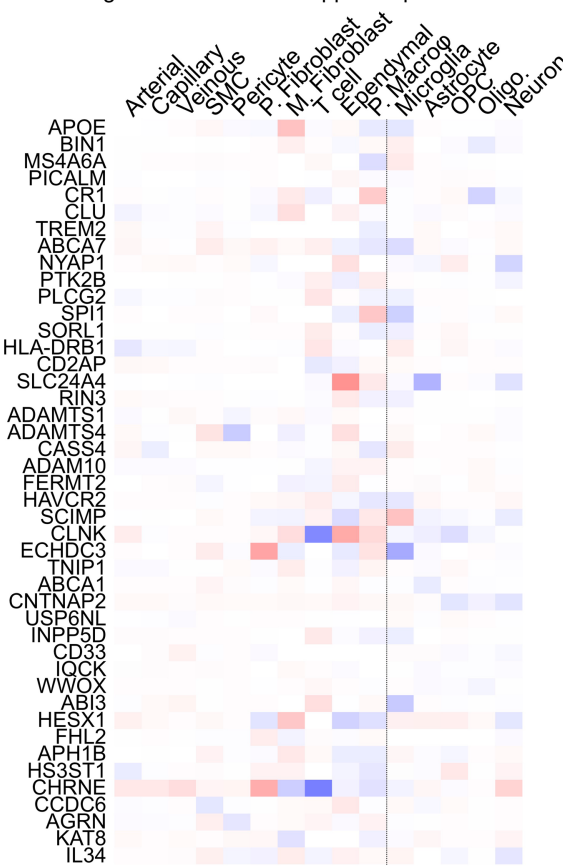

Hpc - Ctx expression

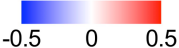

b Minimal expression of T-cell-specific ABCA7 and CLNK among human brain parenchymal cells (Zhang, et al. 2016)

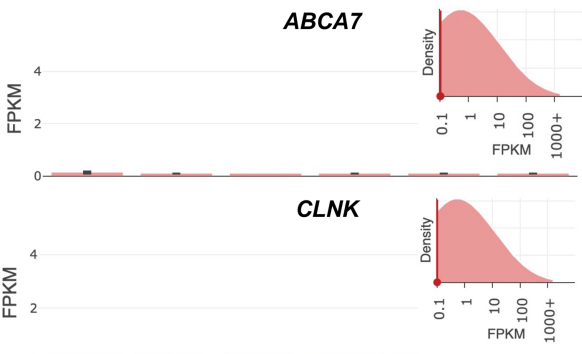

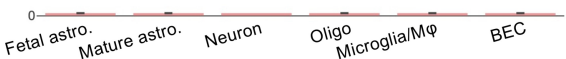

C Brain vascular localization of top AD GWAS proteins

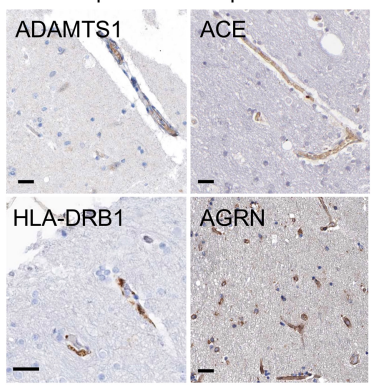

e Microglia-specific GWAS genes (Grubman, et al. 2019) are also expressed in vascular cells
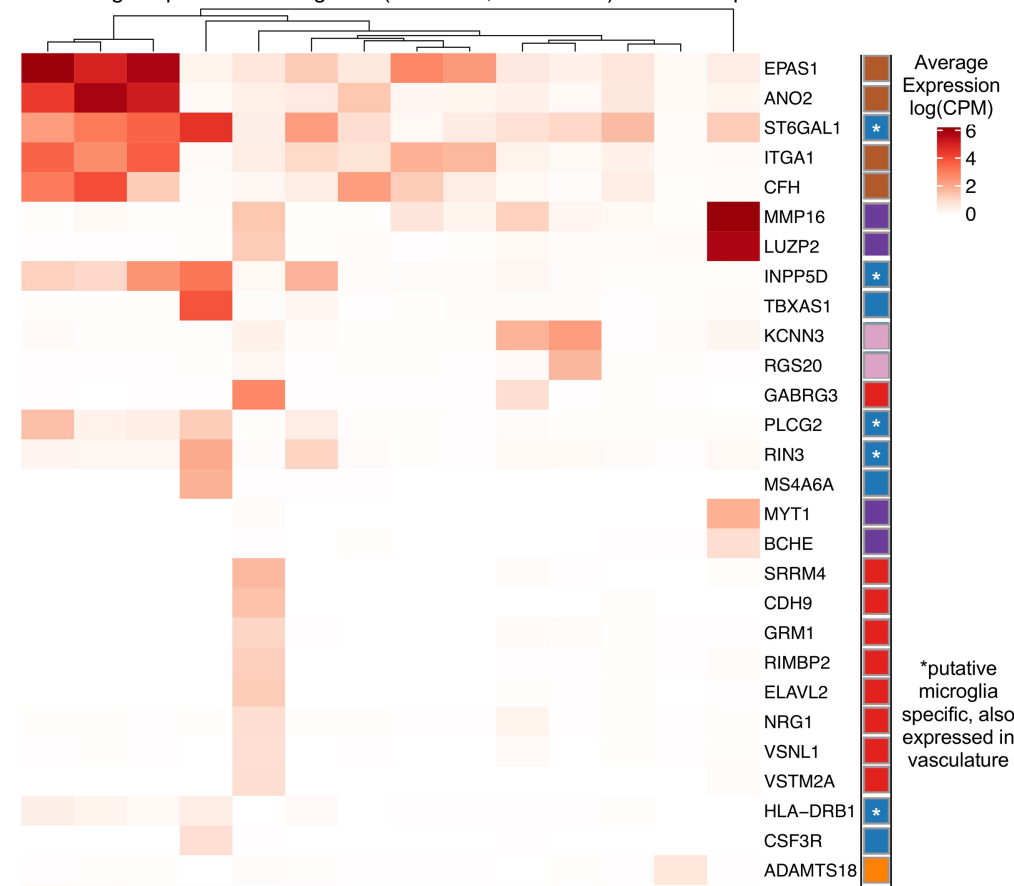

LUZP2

INPP5D

TBXAS1

KCNN3

RGS20

GABRG3

PLCG2

RIN3

MS4A6A

MYT1

BCHE

SRRM4

CDH9

GRM1

RIMBP2

ELAVL2

NRG1

VSNL1

VSTM2A

HLA-DRB1 *

CSF3R

ADAMTS18

HLA-DRB5 *

$\mathrm{PCDH8}$

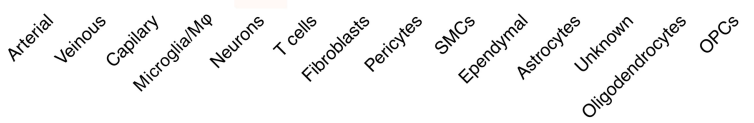

f Human vascular-microglia axis mediating protein transport/ clearance \& inflammation

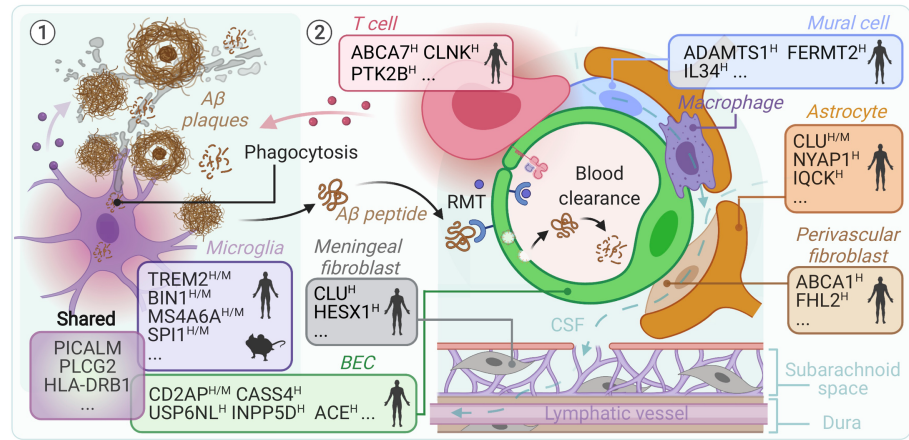

Extended Data Fig. $9 \mid$ See next page for caption. 
Extended Data Fig. 9 | Re-evaluation and characterization of top AD GWAS genes expressed in the human brain vasculature. a, Heterogeneous

expression of AD GWAS genes across T- and M-pericyte subtypes. b, RNA-seq data of the predicted T cell-specific AD GWAS genes EPHA1 and $A B C A 7$ in an independent dataset ${ }^{81}$, corroborating minimal expression across resident/ parenchymal brain cells. c, Immunohistochemical confirmation of vascular localization of proteins encoded by 12 top AD GWAS genes from a. Scale bars, $25 \mu \mathrm{m}$. Arrowheads in APOE point to signal around larger-diameter vessels, consistent with predicted SMC expression. Image credit: Human Protein Atlas $^{25,74}$ (http://www.proteinatlas.org).d, Heat map comparing expression patterns of top AD GWAS genes in the hippocampus and superior frontal cortex: e.g., several microglia-expressed GWAS genes like $A P O E, M S 4 A 4 A$, and TREM2 are more highly expressed in hippocampal compared to cortical microglia/macrophages. e, GWAS genes found to be expressed specifically in microglia among cells captured using the conventional nuclei isolation process (from Grubman et al. 2019) ${ }^{17}$ are also expressed in vascular cells (asterisks). f, Summary of AD GWAS genes enriched in microglia and vascular cells mediating common pathways in protein clearance and inflammation. Mouse and human superscripts denote whether expression has been confirmed in that species for a given gene. Proposed model is described in Discussion. 
a Robust expression of AD GWAS genes across vascular cell types $(\log C P M>2,>10 \%)$

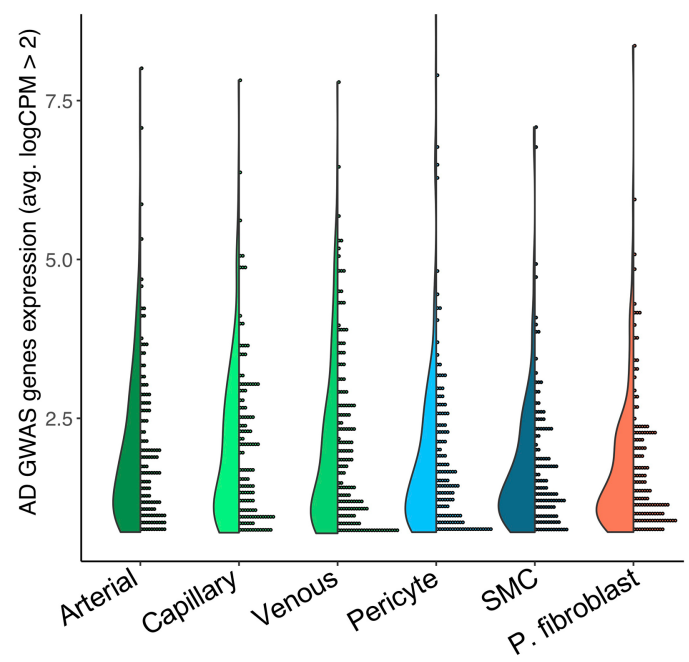

b Example enriched pathways in $A D$ \& AD-related trait GWAS genes across human brain cell types

\begin{tabular}{l} 
Endothelial \\
$-\log _{10}(P$-value $)$ \\
\begin{tabular}{llll}
\hline & 2 & 3 & 4
\end{tabular} \\
\hline IL-5 reg. of apoptosis \\
TNF-a effects \\
Leptin influ. on immune resp. \\
Cell adhesion molecules \\
Hemostasis
\end{tabular}

\begin{tabular}{l} 
Pericyte \& SMC \\
$-\log _{10}(P$-value $)$ \\
\hline Gas signaling \\
Fibroblast growth factor 1 \\
p53 activity regulation \\
Calcium reg. in cardiac cell \\
Myometrial relaxation \\
\& contraction
\end{tabular}
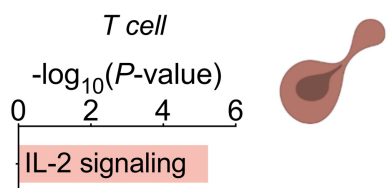

RANKL reg. of immune resp.

TNFa effects

IL-7 signaling

Chemokine signaling

$$
\begin{aligned}
& \text { Microglia \& } M \varphi \\
& -\log _{10}(P \text {-value }) \\
& \begin{array}{lllll}
0 & 1 & 2 & 3 & 4 \\
\hline
\end{array} \\
& \text { TGF- } \beta \text { signaling } \\
& \text { Immune system } \\
& \text { Interleukin receptor SHC signaling } \\
& \text { Lipid and lipoprotein metabolism } \\
& \text { IL-3, IL-5, GM-CSF signaling }
\end{aligned}
$$

Extended Data Fig. 10 | Brain vascular and perivascular expression of AD and AD-related GWAS genes. a, Expression of AD and AD-related GWAS risk genes (from Grubman et al. 2019) ${ }^{17}$ across human vascular cells. b, Enriched
C Example cell type-specific AD \& AD-related trait GWAS hits

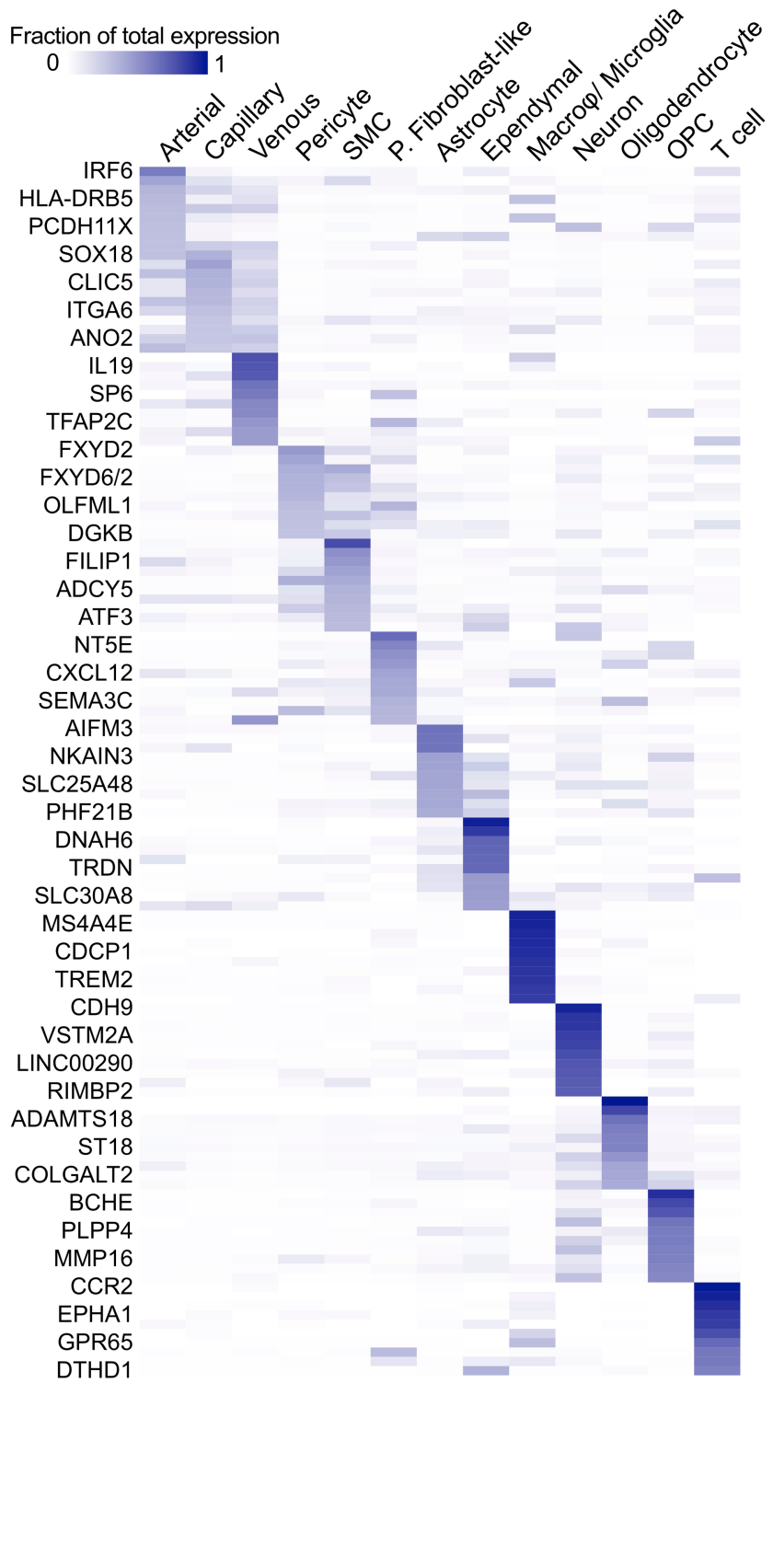

biological pathways amongst AD and AD-related trait GWAS genes expressed in each cell type.c, For each cell type, the top 10 most specifically expressed AD and AD-related trait GWAS genes. 


\section{Reporting Summary}

Nature Research wishes to improve the reproducibility of the work that we publish. This form provides structure for consistency and transparency in reporting. For further information on Nature Research policies, see our Editorial Policies and the Editorial Policy Checklist.

\section{Statistics}

For all statistical analyses, confirm that the following items are present in the figure legend, table legend, main text, or Methods section.

$\mathrm{n} / \mathrm{a}$ Confirmed

$\bigotimes$ The exact sample size $(n)$ for each experimental group/condition, given as a discrete number and unit of measurement

$\triangle$ A statement on whether measurements were taken from distinct samples or whether the same sample was measured repeatedly

The statistical test(s) used AND whether they are one- or two-sided

Only common tests should be described solely by name; describe more complex techniques in the Methods section.

$\square$ A description of all covariates tested

$\square$ \ A description of any assumptions or corrections, such as tests of normality and adjustment for multiple comparisons

$\square$ A full description of the statistical parameters including central tendency (e.g. means) or other basic estimates (e.g. regression coefficient)

AND variation (e.g. standard deviation) or associated estimates of uncertainty (e.g. confidence intervals)

$\square$ For null hypothesis testing, the test statistic (e.g. $F, t, r$ ) with confidence intervals, effect sizes, degrees of freedom and $P$ value noted

Give $P$ values as exact values whenever suitable.

Х $\square$ For Bayesian analysis, information on the choice of priors and Markov chain Monte Carlo settings

Х $\square$ For hierarchical and complex designs, identification of the appropriate level for tests and full reporting of outcomes

Х $\square$ Estimates of effect sizes (e.g. Cohen's $d$, Pearson's $r$ ), indicating how they were calculated

Our web collection on statistics for biologists contains articles on many of the points above.

\section{Software and code}

Policy information about availability of computer code

Data collection For droplet-based snRNA-seq, libraries were prepared using the Chromium Next GEM Single Cell 3' v3.1 according to the manufacturer's protocol (10x Genomics), targeting 10,000 nuclei per sample after counting with a TC20 Automated Cell Counter (Bio-Rad). Generated snRNAseq libraries were sequenced across S4 lanes on a NovaSeq 6000 (150 cycles, Novogene).

Data analysis Gene counts were obtained by aligning reads to the hg38 genome (refdata-gex-GRCh38-2020-A) using CellRanger software (v.4.0.0) (10x Genomics). Data were primarily analyzed using the Seurat 3.0 package in R (v.3.6.3). Specific analysis also used the packages: Monocle3, CellChat (v.0.02), Enrichr, Metascape, GeneTrail 3, dplyr, dplyr (v.1.0.0), ggplot2 (v.3.2.2.), patchwork (v.1.0.1), openxlsx (v.4.1.5), bioconductor-scater (v.1.14.6), bioconductor-dropletutils (v1.6.1), bioconductor-complexheatmap (v.2.2.0), tidyverse (v.1.3.0), and Isa (v.0.73.2). All other tasks were performed on an x86_64-based Ubuntu server cluster.

For manuscripts utilizing custom algorithms or software that are central to the research but not yet described in published literature, software must be made available to editors and

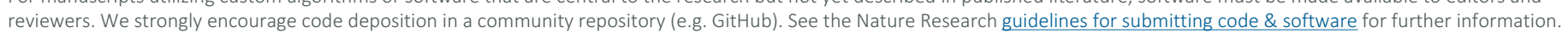

\section{Data}

Policy information about availability of data

All manuscripts must include a data availability statement. This statement should provide the following information, where applicable:

- Accession codes, unique identifiers, or web links for publicly available datasets

- A list of figures that have associated raw data

- A description of any restrictions on data availability

Raw sequencing data in this study has been deposited in NCBI GEO: GSE163577. Data is also available to explore via an interactive web browser: https://twc-stanford.shinyapps.io/human_bbb. We reference previously published mouse sequencing data deposited in NCBI GEO: GSE98816 and GSE99058. 
Please select the one below that is the best fit for your research. If you are not sure, read the appropriate sections before making your selection.

\ Life sciences

Behavioural \& social sciences

Ecological, evolutionary \& environmental sciences

For a reference copy of the document with all sections, see nature.com/documents/nr-reporting-summary-flat.pdf

\section{Life sciences study design}

All studies must disclose on these points even when the disclosure is negative.

Sample size We did not use statistical methods to pre-determine sample sizes, but our sample sizes are similar to those reported in previous publications. Sample size was primarily determined by the availability of high-quality tissue that was dissected consistently for each brain region.

Data exclusions For all single-nucelus sequencing experiments, low-quality nuclei were excluded using standard, pre-established approaches: (1) ambient cell free mRNA contamination was removed using SoupX for each individual sample; (2) outliers with a high ratio of mitochondrial $(>5 \%,<200$ features) relative to endogenous RNAs and homotypic doublets (> 5000 features) were removed in Seurat; and (3) after scTransform normalization and integration, doublets and multiplets were filtered out using DoubletFinder with subsequent manual inspection and filtering based on cell type-specific marker genes.

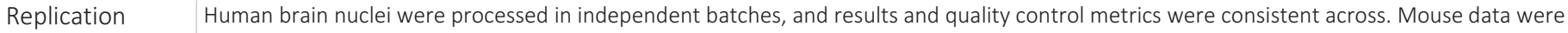
repeated once and successfully replicated.

Randomization For human brain nuclei, equal representation of control and AD samples were ensured in each batch of nuclei isolation. The exact samples from each disease group were then randomly selected for processing in each batch. Human subjects are matched for age, sex, and other covariates. For mouse brain nuclei, mice were randomly chosen within each age group, young (3 month old) or aged (19 month old).

Blinding Investigators were blinded to sample groups during nuclei isolation steps. Single-nucleus RNA-sequencing was unblinded after pre-established quality control and integration steps (e.g., see "Data Exclusions").

\section{Reporting for specific materials, systems and methods}

We require information from authors about some types of materials, experimental systems and methods used in many studies. Here, indicate whether each material, system or method listed is relevant to your study. If you are not sure if a list item applies to your research, read the appropriate section before selecting a response.

\begin{tabular}{l|l} 
Materials \& experimental systems \\
\hline$n / a$ & Involved in the study \\
$\square$ & $\square$ Antibodies \\
$\square$ Eukaryotic cell lines \\
$\square$ Palaeontology and archaeology \\
$\square$ Animals and other organisms \\
$\square$ Human research participants \\
$\square$ \\
$\square$ Clinical data
\end{tabular}

\begin{tabular}{l} 
Methods \\
\hline n/a \\
$\square$ \\
$\square$ Involved in the study \\
$\square$ ChIP-seq \\
$\square$ Flow cytometry \\
$\square$ MRI-based neuroimaging
\end{tabular}

Antibodies

Antibodies used Goat polyclonal anti-Collagen Type IV (1:200, AB769, Sigma), rabbit polyclonal anti-CYP1B1 (1:100, HPA026863, Atlas Antibodies), rabbit polyclonal anti-SLC4A4 (1:100, HPA035628, Atlas Antibodies), rabbit polyclonal anti-SLC47A1 (1:100, HPA021987, Atlas Antibodies), rabbit polyclonal anti-ABCA8 (1:100, HPA044914, Atlas Antibodies), mouse monoclonal anti-CD31 (1:100, JC70A, Dako), rabbit polyclonal anti-VWF (1:100, GA527, Dako), rabbit polyclonal anti-SLC39A10 (1:100, HPA066087, Atlas Antibodies), rabbit polyclonal anti-ALPL (1:100, HPA007105, Atlas Antibodies), rabbit polyclonal anti-A2M (1:100, HPA002265, Atlas Antibodies), rabbit monoclonal anti- $\beta$-Amyloid (1:500, clone D54D2 XP, CST 8243T), and mouse monoclonal anti-Actin, $\alpha$-Smooth Muscle - Cy3 (1:100, clone 1A4, Sigma C6198).

Immunostaining secondary antibodies: Alexa Fluor 488 donkey anti-goat-IgG (1:250; Invitrogen, A-11055); Alexa Fluor 488 donkey anti-rat-IgG (1:250; Invitrogen, A-21208); Alexa Fluor 555 donkey anti-mouse-IgG (1:250; Invitrogen, A-31570); Alexa Fluor 555 donkey anti-goat-lgG (1:250; Invitrogen, A-21432); Alexa Fluor 647 donkey anti-mouse-lgG (1:250; Invitrogen, A-31571); Alexa Fluor 647 donkey anti-rabbit-IgG (1:250; Invitrogen, A-31573); Alexa Fluor 647 donkey anti-goat-IgG (1:250; Invitrogen, A-21447); Alexa Fluor 647 donkey anti-rabbit-IgG (1:250; Invitrogen, A-31573); and Alexa Fluor 647 donkey anti-goatlgG (1:250; Invitrogen, A-21447). 


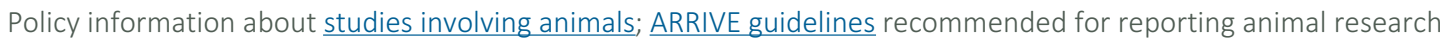

Laboratory animals

Wild animals

Field-collected samples

Ethics oversight

\section{Human research participants}

Policy information about studies involving human research participants

Population characteristics

Recruitment

Ethics oversight
Male and female aged (58-93 years old) cognitively normal and clinically-diagnosed Alzheimer's disease patients. Cognitively normal patients do not display atypical vascular pathologies. Patients are mixed in APOE genotype.

Subjects were not recruited specifically for this study. Samples are derived from a brain bank maintained by the Stanford/ VA/ NIA Aging Clinical Research Center (ACRC) from patients that provide consent for broad, de-identified data sharing under Institutional Review Board (IRB) approval.

Stanford/ VA/ NIA Aging Clinical Research Center (ACRC)

Note that full information on the approval of the study protocol must also be provided in the manuscript. 